\title{
Indifference Pricing and Hedging in a Multiple-Priors Model with Trading Constraints*
}

\author{
Huiwen Yan ${ }^{\dagger}$ Gechun Liang ${ }^{\ddagger}$ Zhou Yang $\S$
}

\begin{abstract}
This paper considers utility indifference valuation of derivatives under model uncertainty and trading constraints, where the utility is formulated as an additive stochastic differential utility of both intertemporal consumption and terminal wealth, and the uncertain prospects are ranked according to a multiple-priors model of Chen and Epstein (2002). The price is determined by two optimal stochastic control problems (mixed with optimal stopping time in the case of American option) of forward-backward stochastic differential equations. By means of backward stochastic differential equation and partial differential equation methods, we show that both bid and ask prices are closely related to the Black-Scholes risk-neutral price with modified dividend rates. The two prices will actually coincide with each other if there is no trading constraint or the model uncertainty disappears. Finally, two applications to European option and American option are discussed.
\end{abstract}

Key words: Indifference pricing, stochastic differential utility, trading constraints, ambiguity, variational inequality, American option.

AMS subject classifications (2000): 35R60, 47J20, 93E20

\section{Introduction}

This paper considers derivative pricing in an incomplete financial market with model uncertainty (ambiguity) and trading constraints. Incompleteness means that investors are uncertain about the riskneutral probability measure which are used to price the derivatives in the market. The investors rank the uncertain prospects according to a multiple-priors model of Chen and Epstein [4, where a continuoustime intertemporal version of multiple-priors utility is formulated by using backward stochastic differential equation (BSDE). The other source of incompleteness is due to trading constraints such as short sale constraint. We take the indifference pricing approach where the investor's utility is formulated as an additive stochastic differential utility of both intertemporal consumption and terminal wealth (see Duffie and Epstein [8]).

The idea of indifference valuation is firstly introduced by Hodges and Neuberger [15, whereby the price for a derivative is the cash amount that the investor is willing to pay such that she is no worse off in

\footnotetext{
* The work is supported by NNSF of China (No.11271143, 11371155), University Special Research Fund for Ph.D. Program (No. 20124407110001), NNSF of Zhejiang Province(No. Y6110775), and the Oxford-Man Institute of Quantitative Finance. We thank two anonymous referees for their valuable comments and helpful suggestions on our paper.

${ }^{\dagger}$ School of Mathematics and Computer Science, Guangdong University of Finance \& Economics, Guangzhou 510320, China, hwyan10@gmail.com

‡Department of Mathematics, King’s College London, London WC2R 2LS, U.K., gechun.liang@kcl.ac.uk

$\S$ School of Mathematical Science, South China Normal University, Guangzhou 510631, China, yangzhou@scnu.edu.cn
} 
expected utility terms than she would have been without the derivative. The idea is further developed by Henderson [13, Musiela and Zariphopoulou [23] and Sicar and Zariphopoulou [24, among others, under a Markovian setting using the partial differential equation (PDE) approach, and by Hu et al [16, Mania and Schweizer 22] and Ankirchner et al 2 in a general non-Markovian setting using the BSDE approach (see more references therein). On the other hand, Becherer [3] and Davis [7] use the duality approach to study the indifference hedging strategy. However, most of the existing research is based on the assumption that the investor is only concerned with her terminal wealth, and ignores her intertemporal consumption and her risk aversion in the consumption. It seems that the only exception is Cheridito and $\mathrm{Hu}[\mathbf{5}$, which takes account of consumption on the top of terminal wealth under the framework of Hu et al [16. In our model, the investor takes account of not only the terminal wealth but also the intertemporal consumption, and she is risk averse in the consumption instead of the terminal wealth.

Different from most of utility indifference pricing models, where the indifference prices are heavily distorted by the investor's risk aversion in her terminal wealth, and therefore complicated in general, the indifference prices in our model are independent of the investor's risk aversion, and bear a striking resemblance to the risk-neutral price. In fact, we show that both bid and ask prices in our model are closely related to the risk-neutral price with modified dividend rates. The deviation is actually caused by the investor's uncertainty on the risk-neutral probability measure and the existence of the trading constraints.

Model uncertainty is an important aspect in derivative pricing. Indeed, uncertainty on the choice of an option pricing model can lead to model risk in the valuation of portfolios of options, so one must separate between risk (uncertainty on outcomes for which the probabilities are known) and ambiguity (model uncertainty). In their seminal work, Chen and Epstein [4 introduce a multiple-priors model under the framework of stochastic differential utility. Cont [6] introduces different risk measures to quantity the model uncertainty. In the indifference pricing framework, Jaimungal and Sigloch [17] introduce the concept of robust indifference pricing (with the utility of terminal wealth), which incorporates both risk aversion and model uncertainty. They mainly use the idea from Anderson et al [1, by modifying the optimization problem to maximize the expected penalized utility of terminal wealth, while minimizing the expected penalized utility over a set of equivalent measures. Since the risk-neutral probability measure is naturally a dominant pricing measure, we take the multiple-priors model from Chen and Epstein [4] in order to incorporate the model uncertainty, where the probability measures in the set of priors are equivalent to the risk-neutral dominant pricing measure.

The idea of applying Chen and Epstein's multiple-priors model to derivative pricing is not new. For example, Guo et al [12] consider the pricing problem of exotic options, in particular Parisian options, also under the multiple-priors framework of Chen and Epstein [4. However, they use the idea of superreplication rather than utility indifference valuation, so they obtain pricing bounds rather than a price. On the other hand, their concentration is more on numerical solutions of the pricing bounds, which is different from our paper where we try to build up an indifference pricing framework under model uncertainty. Moreover, our model also includes the trading constraints on the top of model uncertainty. Actually, these two factors result in the market incompleteness in our model.

Although the model uncertainty causes the deviation of the bid and ask prices from the risk-neutral price, we show that if there is no trading constraint, then both bid and ask prices will coincide with the risk-neutral price even under model uncertainty. In fact, if there is no trading constraint, the investor can invest arbitrary position in the underlying stocks to hedge the model uncertainty in our indifference pricing setup. On the other hand, if there exist trading constraints, then the prices will deviate from the risk-neutral price. For example, if the payoff of an option is monotone with respect to the prices of the 
underlying, the investor needs to hold an opposite position in order to hedge her risk exposure. Trading constraints such as short sale constraint will prohibit her arbitrary position, and therefore result in prices different from the risk-neutral price. We should remark that the price deviation is not only caused by trading constraints but also model uncertainty. These two factors tangling together impact the bid and ask prices, in both European option and American option cases. We also obtain the convergence rate of the indifference prices to the risk-neutral price as the model uncertainty disappears (see Proposition 3.5 for the case of European option and Proposition 4.8 for the case of American option).

If the investor is allowed to exercise her option at any time before maturity as in the American option setting, the definition of the corresponding indifference price needs to be modified. In such a situation, the investor needs to compare two optimal investment problems with different time horizons, and she chooses not only her optimal trading strategy but also her optimal exercise time. By the time consistency, we derive an intertemporal wealth which consists of not only the usual wealth, but also the value of the remaining optimal consumption (see Definition 4.2). In a Markovian setting, we also effectively use the Alexandrov-Bakel'man-Pucci (A-B-P) comparison principle for variational inequalities to deduce various properties of the bid and ask prices for American option.

The utility indifference pricing models are usually formulated as two optimal portfolio problems (see (2.7) and (2.8) ). Concretely speaking, the price $P$ is determined from an equation $F(\cdot, P(\cdot))=G(\cdot)$, and functions $F$ and $G$ where the value functions of two optimal stochastic control problems. There are two methods to solve these problems, such as the BSDE approach and the PDE approach. The BSDE approach is based on martingale optimality principle (see [16, 22]) or the risk-sensitive control (see a recent work by Henderson and Liang [14), and the price is expressed via the solutions of two BSDEs. The PDE method is based on dynamic programming principle, and the price is described via the solutions of two HJB equations (see [13, 23, 24]). Due to the complexities of BSDEs and HJB equations, it is usually difficult to investigate the properties of the utility indifference prices.

In our model, the price is still determined by the equation $F(\cdot, P(\cdot))=G(\cdot)$, where $F$ and $G$ are the value functions of the two optimal control problems of forward-backward stochastic equations (FBSDEs). In the American option case, the optimal control problems involve optimal stopping time problems, and the optimal strategy consists of optimal consumption, optimal investment and optimal stopping time.

In order to completely solve the problems, we firstly analyze the two optimal portfolio problems and express the price as the solution of nonlinear BSDEs via the following idea. We define an indirect utility by subtracting the wealth (and the contingent claim) from the original stochastic differential utility (SDU) to represent the indifference price as the solution of BSDE with Lipschitz continuous driver. Mathematically speaking, we translate the original stochastic control problem of FBSDE into finding a maximum solution to a family of BSDEs with different drivers. Then by the comparison principle for BSDE, we find the nonlinear BSDE for the optimal solution and express the price via the corresponding FBSDE.

By applying the Feynman-Kac formula, we express the prices as the solutions of quasi-linear PDE (for European option) or variational inequality (VI, for American option). Then via the method of PDE, we improve the regularity of the value function, and analyze the properties of the price, the optimal investment, consumption and stopping strategies. In this paper, We give a general and technical proof of improving the regularity of the solution of PDE or VI under low regularity of terminal value and obstacles. Moreover, we prove that PDE is a special case of VI if we choose a proper obstacle under some general assumptions. Thanks to the improved regularity, we achieve some concise results as mentioned above. With these results, it is easy to calculate the prices or theoretically analyze their properties by the standard method. Moreover, we use some PDE estimates to show the convergence result of the corresponding prices when the ambiguity market shrinks to the standard market. 
The paper is organized as follows: We present our indifference pricing and hedging model in Section 2, and apply it to two concrete settings, namely European option and American option in Section 3 and Section 4 respectively. Some further technical details on the results of relevant PDEs are provided in the Appendix.

\section{Indifference Pricing and Hedging Model}

For a fixed time horizon $T>0$, let $W=\left(W^{1}, \cdots, W^{n}\right)^{T}$ be an $n$-dimensional Brownian motion on a filtered probability space $\left(\Omega, \mathcal{F}, \mathbb{F}=\left\{\mathcal{F}_{t}\right\}, \mathbb{P}\right)$ satisfying the usual conditions, where $\mathbb{F}$ is the augmented filtration generated by the Brownian motion $W$, and $\mathbb{P}$ is interpreted as the risk-neutral probability measure. Herein the superscript $T$ denotes the matrix transposition. The market consists of one risk-free asset $B$ with the risk-free interest rate $r(\cdot)$, and $n$ risky assets $S=\left(S^{1}, \cdots, S^{n}\right)^{T}$, whose price processes under the risk-neutral probability measure $\mathbb{P}$ are given by

$$
S_{s}^{i}=S_{t}^{i}+\int_{t}^{s} r(u) S_{u}^{i} d u+\sum_{j=1}^{n} \int_{t}^{s} \sigma_{i j}(u) S_{u}^{i} d W_{u}^{j},
$$

for $1 \leq i \leq n$ and $0 \leq t \leq s \leq T$, where $\sigma(\cdot)=\left(\sigma_{i j}(\cdot)\right)_{1 \leq i, j \leq n}$ is the volatility matrix. The coefficients satisfy the following assumption:

Assumption 2.1 The risk-free interest rate $r(\cdot)$ and the volatility matrix $\sigma(\cdot)$ are continuous functions, and the volatility matrix $\sigma(\cdot)$ is positive definite.

However, investors are uncertain about the risk-neural probability measure $\mathbb{P}$, and rank the uncertain prospects according to a multiple-priors model, which was initially proposed by Chen and Epstein 4. They represent the set $\Theta$ of priors on $\left(\Omega, \mathcal{F}_{T}\right)$ by the set of probability measures equivalent to $\mathbb{P}$ :

$$
\Theta \triangleq\left\{\mathbb{Q}: \frac{d \mathbb{Q}}{d \mathbb{P}}=\exp \left(-\frac{1}{2} \int_{0}^{T}\left|\xi_{s}\right|^{2} d s-\int_{0}^{T}\left(\xi_{s}\right)^{T} d W_{s}\right)\right\},
$$

for $\xi=\left(\xi^{1}, \cdots, \xi^{n}\right)^{T} \in \Xi$, where $\Xi$ is the set of $\mathbb{F}$-adapted process valued in a compact and convex subset $\mathcal{O} \subset \mathbb{R}^{n}$ including the origin 0 . More generally, the density on $\left(\Omega, \mathcal{F}_{t}\right)$ is defined as $\mathbb{E}_{\mathbb{P}}\left[\frac{d \mathbb{Q}}{d \mathbb{P}} \mid \mathcal{F}_{t}\right]$. Hence, $\Theta$ is indeed the set of equivalent probability measures which includes the risk-neutral probability measure $\mathbb{P}$ as the dominant pricing measure.

For any starting time $t \in[0, T]$, a representative investor makes inter-temporal consumption and invests the remaining wealth in the risk-free asset and the risky assets in the remaining time interval $[t, T]$, resulting in her wealth equation:

$$
\begin{aligned}
X_{s}^{X_{t} ; \pi, c} & =X_{t}+\int_{t}^{s} \frac{X_{u}^{X_{t} ; \pi, c}-\sum_{i=1}^{n} \pi_{u}^{i}}{B_{u}} d B_{u}+\sum_{i=1}^{n} \int_{t}^{s} \frac{\pi_{u}^{i}}{S_{u}^{i}} d S_{u}^{i}-\int_{t}^{s} c_{u} d u \\
& =X_{t}+\int_{t}^{s}\left[r(u) X_{u}^{X_{t} ; \pi, c}-c_{u}\right] d u+\int_{t}^{s}\left(\pi_{u}\right)^{T} \sigma(u) d W_{u}
\end{aligned}
$$

where $(\pi, c)$ is the portfolio-consumption strategy with $c$ being the inter-temporal consumption rate, and $\pi=\left(\pi^{1}, \cdots, \pi^{n}\right)^{T}$ being the amount of money invested in the risky assets $S=\left(S^{1}, \cdots, S^{n}\right)^{T}$, both in the admissible set $\Pi[t, T]$ :

$$
\Pi[t, T] \triangleq\left\{(\pi, c): \pi \in L_{\mathbb{F}}^{2}(t, T ; \mathcal{A}), c \in L_{\mathbb{F}}^{2}\left(t, T ; \mathbb{R}_{+}\right)\right\}
$$


with

$$
L_{\mathbb{F}}^{2}(t, T ; \mathcal{A}) \triangleq\left\{\pi: \mathbb{F} \text {-adapted, valued in } \mathcal{A}, \text { and } \mathbb{E}_{\mathbb{P}}\left[\int_{t}^{T}\left|\pi_{s}\right|^{2} d s\right]<\infty\right\},
$$

where $\mathcal{A}$ is a closed subset of $\mathbb{R}^{n}$.

Note that the investor in fact makes her decision under $\mathbb{Q}$ but not under $\mathbb{P}$, as she is uncertain about the risk-neutral probability $\mathbb{P}$. By Girsanov's theorem, $\bar{W}=\left(\bar{W}^{1}, \cdots, \bar{W}^{n}\right)^{T}$ with $\bar{W}^{j}=W^{j}+\int_{0}^{\cdot} \xi_{s}^{j} d s$ is the Brownian motion under $\mathbb{Q}$, and the investor's wealth equation (2.2) under $\mathbb{Q}$ is

$$
\begin{aligned}
X_{s}^{X_{t} ; \pi, c} & =X_{t}+\int_{t}^{s}\left[r(u) X_{u}^{X_{t} ; \pi, c}-c_{u}-\sum_{i, j=1}^{n} \sigma_{i j}(u) \pi_{u}^{i} \xi_{u}^{j}\right] d u+\sum_{i, j=1}^{n} \int_{t}^{s} \sigma_{i j}(u) \pi_{u}^{i} d \bar{W}_{u}^{j} \\
& =X_{t}+\int_{t}^{s}\left[r(u) X_{u}^{X_{t} ; \pi, c}-c_{u}-\left(\pi_{u}\right)^{T} \sigma(u) \xi_{u}\right] d u+\int_{t}^{s}\left(\pi_{u}\right)^{T} \sigma(u) d \bar{W}_{u} .
\end{aligned}
$$

Hence, the portfolio-consumption strategy $(\pi, c)$ does impact the investor's wealth $X^{X_{t} ; \pi, c}$ through its drift terms.

The investor has an additive utility defined on the priors set $\Theta$, which is formulated as a stochastic differential utility as in Duffie and Epstein [8]:

$$
U_{t}^{\mathbb{Q}} \triangleq \mathbb{E}_{\mathbb{Q}}\left[\int_{t}^{\tau}\left(v\left(u, c_{u}\right)-r(u) U_{u}^{\mathbb{Q}}\right) d u+U_{\tau}^{\mathbb{Q}} \mid \mathcal{F}_{t}\right],
$$

with the bequest at the terminal time $T: U_{T}^{\mathbb{Q}}=X_{T}^{X_{t} ; \pi, c}$, where $\tau \in \mathcal{U}[t, T]$ is the set of $\mathbb{F}$-stopping times valued in $[t, T], v(\cdot, \cdot)$ is the time-dependent utility of the inter-temporal consumption rate $c$, and $X^{X_{t} ; \pi, c}$ is the wealth process. The investor chooses the worst scenario from the priors set $\Theta$ as her utility:

$$
U_{t}=\inf _{\mathbb{Q} \in \Theta} U_{t}^{\mathbb{Q}} .
$$

The utility $v(\cdot, \cdot)$ of the consumption rate $c$ satisfies the following assumption:

Assumption 2.2 For any $t \in[0, T]$, the time-dependent utility $v:[0, T] \times \mathbb{R} \mapsto[-\infty, \infty)$ is (1) concave, nondecreasing and upper semi-continuous; (2) the half-line $\operatorname{dom}_{t}(v) \triangleq\{x \in[0,+\infty): v(t, x)>-\infty\}$ is a nonempty subset of $[0, \infty)$; and (3) $\partial_{x} v(t, \cdot)$ is continuous, positive, and strictly decreasing in the interior of $\operatorname{dom}_{t}(v)$, and

$$
\lim _{x \rightarrow+\infty} \sup _{t \in[0, T]} \partial_{x} v(t, x)=0 .
$$

A typical example of $v(\cdot, \cdot)$ is the power utility which is penalized to $-\infty$ when the consumption rate is negative. By Section 3.4 of Karatzas and Shreve [19], there exists $\widehat{c}(t)$ such that

$$
v^{*}(t) \triangleq v(t, \widehat{c}(t))-\widehat{c}(t)=\sup _{x \in \mathbb{R}}\{v(t, x)-x\} .
$$

That is, $v^{*}(t)$ is the convex dual of $v(t, \cdot)$ at level 1 for $t \in[0, T]$.

The investor values a contingent claim in the market, whose payoff is an $\mathcal{F}_{T}$-measurable random variable $\xi \in L_{\mathcal{F}_{T}}^{2}\left(\mathbb{R}_{+}\right)$:

$$
L_{\mathcal{F}_{T}}^{2}\left(\mathbb{R}_{+}\right) \triangleq\left\{\xi: \mathcal{F}_{T^{-}} \text {-measurable, valued in } \mathbb{R}_{+} \text {, and } \mathbb{E}_{\mathbb{P}}\left[|\xi|^{2}\right]<\infty\right\} .
$$

If there is no ambiguity about the risk-neutral probability measure $\mathbb{P}$, no inter-temporal consumption and no trading constraints, it is known that the risk-neutral price process $D$ of this contingent claim and 
the corresponding hedging strategy $Y=\left(Y^{1}, \cdots, Y^{n}\right)^{T}$ (normalized by the volatility matrix $\left.\sigma(\cdot)\right)$ are the unique solutions to the following linear BSDE:

$$
D_{t}=\xi-\int_{t}^{T} r(u) D_{u} d u-\int_{t}^{T}\left(Y_{u}\right)^{T} d W_{u}=\mathbb{E}_{\mathbb{P}}\left[e^{-\int_{t}^{T} r(u) d u} \xi \mid \mathcal{F}_{t}\right]
$$

for $(D, Y) \in L_{\mathbb{F}}^{2}(0, T ; \mathbb{R}) \times L_{\mathbb{F}}^{2}\left(0, T ; \mathbb{R}^{n}\right)$. See Section 1 of El Karoui et al $[10$ for the further details. However, due to the ambiguity, the consumption and the trading constraints, (2.6) is not valid.

We consider utility indifference valuation for such a contingent claim with the payoff $\xi$. We write the utility $U_{t}$ as $U_{t}\left(X_{T}^{X_{t} ; \pi, c}\right)$, when we want to emphasize the dependence of the utility $U$ on the bequest $X_{T}^{X_{t} ; \pi, c}$.

Definition 2.3 The bid price $P^{b}\left(X_{t} ; \xi\right)$ and the ask price $P^{s}\left(X_{t} ; \xi\right)$ of the contingent claim with the payoff $\xi$ are defined implicitly by the requirement that

$$
\begin{aligned}
& \underset{(\pi, c) \in \Pi[t, T]}{\operatorname{ess.sup}} U_{t}\left(X_{T}^{X_{t} ; \pi, c}\right)=\underset{(\pi, c) \in \Pi[t, T]}{\operatorname{ess.sup}} U_{t}\left(\xi+X_{T}^{X_{t}-P^{b}\left(X_{t} ; \xi\right) ; \pi, c}\right) ; \\
& \underset{(\pi, c) \in \Pi[t, T]}{\operatorname{ess.sup}} U_{t}\left(X_{T}^{X_{t} ; \pi, c}\right)=\underset{(\pi, c) \in \Pi[t, T]}{\operatorname{ess.sup}} U_{t}\left(-\xi+X_{T}^{X_{t}+P^{s}\left(X_{t} ; \xi\right) ; \pi, c}\right),
\end{aligned}
$$

where $X^{X_{t} ; \pi, c}$ follows the wealth equation (2.2) starting from time $t$ under $\mathbb{P}$, or equivalently (2.3) under $\mathbb{Q}$, and $U_{t}(\cdot)$ is the worst case of the stochastic differential utility at time $t$ defined by 2.4), i.e. $U_{t}=$ $U_{t}\left(X_{T}^{X_{t} ; \pi, c}\right)$ satisfies

$$
U_{t}=\inf _{\mathbb{Q} \in \Theta} U_{t}^{\mathbb{Q}}=\inf _{\mathbb{Q} \in \Theta} \mathbb{E}_{\mathbb{Q}}\left[\int_{t}^{T}\left(v\left(u, c_{u}\right)-r(u) U_{u}^{\mathbb{Q}}\right) d u+X_{T}^{X_{t} ; \pi, c} \mid \mathcal{F}_{t}\right] .
$$

In terms of utility maximization, we are thus indifferent between buying or not buying the contingent claim with the payoff $\xi$ for the bid price $P^{b}\left(X_{t} ; \xi\right)$, and indifferent between selling or not selling the contingent claim with the payoff $\xi$ for the ask price $P^{s}\left(X_{t} ; \xi\right)$, while the utility is chosen as the worst scenario from the priors set $\Theta$.

Our model deviates from the existing literature in the following three folds: (1) The utility is formulated in terms of a stochastic differential utility of both the inter-temporal consumption and the terminal wealth, while the most of exiting literature only considers the expected utility of the terminal wealth; (2) The model uncertainty is taken into account in a dynamic consistent way by employing the multiplepriors model of Chen and Epstein [4]; (3) The trading constraints are also considered in our indifference valuation model. We shall see (2) and (3) lead to some new and interesting features of the indifference price.

Our main tool to characterize the indifference price is the theory of BSDE. By Theorem 2.2 of Chen and Epstein [4, the utility $U$ is represented as the unique solution of the following BSDE:

$$
U_{t}=X_{T}^{X_{t} ; \pi, c}+\int_{t}^{T}\left[v\left(u, c_{u}\right)-r(u) U_{u}-\max _{\xi \in \Xi} \sum_{j=1}^{n} \xi_{u}^{j} Z_{u}^{j}\right] d u-\sum_{j=1}^{n} \int_{t}^{T} Z_{u}^{j} d W_{u}^{j}
$$

for $(U, Z) \in L_{\mathbb{F}}^{2}(0, T ; \mathbb{R}) \times L_{\mathbb{F}}^{2}\left(0, T ; \mathbb{R}^{n}\right)$. The BSDEs in this paper are always considered in the above space.

Note that the maximum term in the above bracket is in fact pathwise maximum:

$$
\left(\max _{\xi \in \Xi} \sum_{j=1}^{n} \xi_{t}^{j} Z_{t}^{j}\right)(\omega)=\max _{\xi_{t}(\omega) \in \mathcal{O}} \sum_{j=1}^{n} \xi_{t}^{j}(\omega) Z_{t}^{j}(\omega)
$$


for any $t \in[0, T]$ and $\omega \in \Omega$, so we will write the above two maximization problems synonymously.

Our first main result is the following representation result for the bid price and the ask price.

Theorem 2.4 Suppose that Assumptions 2.1] and 2.2 are satisfied. Then the bid price $P^{b}\left(X_{t} ; \xi\right)$ and the ask price $P^{s}\left(X_{t} ; \xi\right)$ are uniquely determined by Definition 2.3. and are both independent of the initial wealth $X_{t}$. They are denoted as $P^{b}(t ; \xi)$ and $P^{s}(t ; \xi)$ respectively, and have the representations:

$$
\begin{array}{r}
P^{b}(t ; \xi)=\operatorname{ess.sup}_{(\pi, c) \in \Pi[t, T]} U_{t}\left(\xi+X_{T}^{0 ; \pi, c}\right)-\mathcal{R}(t) \leq D_{t}, \\
P^{s}(t ; \xi)=-\operatorname{ess.sup}_{(\pi, c) \in \Pi[t, T]} U_{t}\left(-\xi+X_{T}^{0 ; \pi, c}\right)+\mathcal{R}(t) \geq D_{t},
\end{array}
$$

where $\mathcal{R}(\cdot)$ is the value of the optimal consumption:

$$
\mathcal{R}(t)=\int_{t}^{T} e^{-\int_{t}^{s} r(u) d u} v^{*}(s) d s
$$

Proof. We only consider the case of the bid price, while the case of the ask price is similar. Note that the utility maximization problem on the left hand side (LHS) of (2.7) is a special case of the one on the right hand side (RHS) with $\xi=0$.

We first show that the solutions of both utility maximization problems in (2.7) exist, i.e.

$$
\underset{(\pi, c) \in \Pi[t, T]}{\operatorname{ess.sup}} U_{t}\left(\xi+X_{T}^{X_{t} ; \pi, c}\right)<+\infty
$$

for any payoff $\xi \in L_{\mathcal{F}_{T}}^{2}\left(\mathbb{R}_{+}\right)$and any initial wealth $X_{t} \in L_{\mathcal{F}_{t}}^{2}(\mathbb{R})$. Indeed, if we define an indirect utility $\widehat{U}$ by subtracting the contingent claim $D$ and the wealth $X^{X_{t} ; \pi, c}$ from the original utility $U$ as

$$
\widehat{U}_{s}=U_{s}\left(\xi+X_{T}^{X_{t} ; \pi, c}\right)-\left(D_{s}+X_{s}^{X_{t} ; \pi, c}\right) ; \quad \widehat{Z}_{s}^{j}=Z_{s}^{j}-\left(Y_{s}^{j}+\sum_{i=1}^{n} \sigma_{i j}(s) \pi_{s}^{i}\right)
$$

for $s \in[t, T]$, by (2.2), (2.6) and (2.9), it is easy to verify that $(\widehat{U}, \widehat{Z})$ satisfies the following BSDE:

$$
\widehat{U}_{t}=\int_{t}^{T}\left\{-r(u) \widehat{U}_{u}+\left[v\left(u, c_{u}\right)-c_{u}\right]-\max _{\xi \in \Xi} \sum_{j=1}^{n} \xi_{u}^{j} Z_{u}^{j}\right\} d u-\sum_{j=1}^{n} \int_{t}^{T} \widehat{Z}_{u}^{j} d W_{u}^{j} .
$$

Note that

$$
v\left(u, c_{u}\right)-c_{u} \leq v^{*}(u) ; \quad-\max _{\xi \in \Xi} \sum_{j=1}^{n} \xi_{u}^{j} Z_{u}^{j} \leq 0 .
$$

By the BSDE comparison theorem (see Theorem 2.2 of [10]), $\widehat{U}_{t} \leq \mathcal{R}(t)$, where $\mathcal{R}$ is given by

$$
\mathcal{R}(t)=\int_{t}^{T}\left[-r(u) \mathcal{R}(u)+v^{*}(u)\right] d u-\int_{t}^{T}(\mathcal{Q}(u))^{T} d W_{u}
$$

which has a unique solution in $L_{\mathbb{F}}^{2}(0, T ; \mathbb{R}) \times L_{\mathbb{F}}^{2}\left(0, T ; \mathbb{R}^{n}\right)$ :

$$
(\mathcal{R}(t), \mathcal{Q}(t))=\left(\int_{t}^{T} e^{-\int_{t}^{s} r(u) d u} v^{*}(s) d s, 0\right)
$$

Hence $U_{t}\left(\xi+X_{T}^{X_{t} ; \pi, c}\right) \leq D_{t}+X_{t}+\mathcal{R}(t)$ for any $(\pi, c) \in \Pi$, and we have proved the upper bound:

$$
\underset{(\pi, c) \in \Pi[t, T]}{\operatorname{ess.sup}} U_{t}\left(\xi+X_{T}^{X_{t} ; \pi, c}\right) \leq D_{t}+X_{t}+\mathcal{R}(t)<\infty .
$$


Next, we show the representation (2.10). We first solve the utility maximization problem on LHS of (2.7) explicitly. By taking $(\pi, c)=(0, \widehat{c})$ in (2.9), we have

$$
\widehat{U}_{t}=\int_{t}^{T}\left\{-r(u) \widehat{U}_{u}+v^{*}(u)-\max _{\xi \in \Xi} \sum_{j=1}^{n} \xi_{u}^{j} Z_{u}^{j}\right\} d u-\sum_{j=1}^{n} \int_{t}^{T} Z_{u}^{j} d W_{u}^{j},
$$

where $\widehat{U}_{s} \triangleq U_{s}\left(X_{T}^{X_{t} ; 0, \hat{c}}\right)-X_{s}^{X_{t} ; 0, \widehat{c}}$ for $s \in[t, T]$. The above BSDE has a unique solution in $L_{\mathbb{F}}^{2}(0, T ; \mathbb{R}) \times$ $L_{\mathbb{F}}^{2}\left(0, T ; \mathbb{R}^{n}\right)$, which is the same as (2.13): $\left(\widehat{U}_{t}, Z_{t}\right)=(\mathcal{R}(t), \mathcal{Q}(t))$. Hence

$$
\operatorname{ess.sup}_{(\pi, c) \in \Pi[t, T]} U_{t}\left(X_{T}^{X_{t} ; \pi, c}\right) \geq U_{t}\left(X_{T}^{X_{t} ; 0, \widehat{c}}\right)=\widehat{U}_{t}+X_{t}=\mathcal{R}(t)+X_{t} .
$$

The above inequality is actually the equality. Indeed, By taking $\xi=0$ in (2.14), we have the upper bound:

$$
\operatorname{ess.sup}_{(\pi, c) \in \Pi[t, T]} U_{t}\left(X_{T}^{X_{t} ; \pi, c}\right) \leq \mathcal{R}(t)+X_{t}
$$

so we have proved that

$$
\underset{(\pi, c) \in \Pi[t, T]}{\operatorname{ess.sup}} U_{t}\left(X_{T}^{X_{t} ; \pi, c}\right)=\mathcal{R}(t)+X_{t}
$$

with the optimal portfolio-consumption strategy $\left(\pi^{*}, c^{*}\right)=(0, \widehat{c})$ for the utility maximization problem on LHS of (2.7).

On the other hand, note that the utility maximization problem on RHS of (2.7) is independent of the initial wealth:

$$
\operatorname{ess.sup}_{(\pi, c) \in \Pi[t, T]} U_{t}\left(\xi+X_{T}^{X_{t} ; \pi, c}\right)=\operatorname{ess.sup}_{(\pi, c) \in \Pi[t, T]} U_{t}\left(\xi+X_{T}^{0 ; \pi, c}\right)+X_{t} .
$$

By combining (2.15) and (2.16), we obtain the representation (2.10), which also shows that the bid price $P^{b}(t ; \xi)$ is independent of the initial wealth $X_{t}$. Finally, by taking $X_{t}=0$ in the inequality (2.14) and using the representation (2.10), we obtain the upper bound of the bid price:

$$
P^{b}(t ; \xi)=\underset{(\pi, c) \in \Pi[t, T]}{\operatorname{ess.sup}} U_{t}\left(\xi+X_{T}^{0 ; \pi, c}\right)-\mathcal{R}(t) \leq D_{t}+\mathcal{R}_{t}-\mathcal{R}_{t}=D_{t}
$$

Our next result further characterizes the indifference prices in terms of the solutions to BSDEs. We show that different admissible sets $\Pi$ result in different bid prices $P^{b}(t ; \xi)$ and ask prices $P^{s}(t ; \xi)$, and in particular, if there is no constraint in $\Pi$, then both the bid price and the ask price coincide with the risk-neutral price even with model ambiguity.

For $t \in[0, T]$ and $\omega \in \Omega$, we define the subset $\mathcal{B}_{t}(\omega) \subset \mathbb{R}^{n}$ by

$$
\mathcal{B}_{t}(\omega) \triangleq\left\{z \in \mathbb{R}^{n}: z_{j}=\sum_{i=1}^{n} \sigma_{i j}(t) \pi_{t}^{i}(\omega) \text { with } \pi_{t}(\omega) \in \mathcal{A}\right\} .
$$

Note that $\mathcal{B}_{t}(\omega)$ is still closed since $\mathcal{A}$ is closed and $\sigma_{i j}(\cdot)$ is bounded. For any $\bar{z} \in \mathbb{R}^{n}$, we further introduce

$$
d_{\mathcal{O}}\left(\bar{z}, \mathcal{B}_{t}(\omega)\right)=\min _{z \in \mathcal{B}_{t}(\omega)} \max _{\xi_{t}(\omega) \in \mathcal{O}} \sum_{j=1}^{n} \xi_{t}^{j}(\omega)\left(\bar{z}_{j}+z_{j}\right),
$$

where $\mathcal{O}$ is a closed and convex subset of $\mathbb{R}^{n}$ including the origin 0 . Since $\mathcal{B}_{t}(\omega)$ is closed, there exists at least one point in $\mathcal{B}_{t}(\omega)$ which minimizes the support function $\delta_{\mathcal{O}}(\cdot)$ of the compact and convex set $\mathcal{O}$ :

$$
\delta_{\mathcal{O}}(\bar{z}, z)=\max _{\xi_{t}(\omega) \in \mathcal{O}} \sum_{j=1}^{n} \xi_{t}^{j}(\omega)\left(\bar{z}_{j}+z_{j}\right)
$$


and we denote such minimal point as $\operatorname{argmin}\left(\bar{z}, \mathcal{B}_{t}(\omega)\right)$. In the following, we will omit $\omega$ if no confusion may arise.

Theorem 2.5 Suppose that Assumptions 2.1 and 2.2 are satisfied. Then the bid price $P^{b}(t ; \xi)=U_{t}^{b}$ and the ask price $P^{s}(t ; \xi)=U_{t}^{s}$, where $U^{b}$ and $U^{s}$ are the unique solutions to the following BSDEs respectively:

$$
\begin{aligned}
& U_{t}^{b}=\xi+\int_{t}^{T}\left[-r(u) U_{u}^{b}-d_{\mathcal{O}}\left(Z_{u}^{b}, \mathcal{B}_{u}\right)\right] d u-\int_{t}^{T}\left(Z_{u}^{b}\right)^{T} d W_{u} \\
& U_{t}^{s}=\xi+\int_{t}^{T}\left[-r(u) U_{u}^{s}+d_{\mathcal{O}}\left(-Z_{u}^{s}, \mathcal{B}_{u}\right)\right] d u-\int_{t}^{T}\left(Z_{u}^{s}\right)^{T} d W_{u}
\end{aligned}
$$

for $\left(U^{b}, Z^{b}\right) \in L_{\mathbb{F}}^{2}(0, T ; \mathbb{R}) \times L_{\mathbb{F}}^{2}\left(0, T ; \mathbb{R}^{n}\right)$ and $\left(U^{s}, Z^{s}\right) \in L_{\mathbb{F}}^{2}(0, T ; \mathbb{R}) \times L_{\mathbb{F}}^{2}\left(0, T ; \mathbb{R}^{n}\right)$. The optimal portfolioconsumption strategy for the bid price is

$$
\left(\pi^{*}, c^{*}\right)=\left(\left(\sigma^{T}\right)^{-1} \operatorname{argmin}\left(Z^{b}, \mathcal{B}\right), \widehat{c}\right),
$$

and for the ask price is

$$
\left(\pi^{*}, c^{*}\right)=\left(\left(\sigma^{T}\right)^{-1} \operatorname{argmin}\left(-Z^{s}, \mathcal{B}\right), \widehat{c}\right) .
$$

Moreover, if $\mathcal{A}=\mathbb{R}^{n}$, i.e. there is no trading constraint, then both the bid price $P^{b}(t ; \xi)$ and the ask price $P^{s}(t ; \xi)$ coincide with the risk neutral price $D_{t}$ :

$$
P^{b}(t ; \xi)=P^{s}(t ; \xi)=D_{t}=\mathbb{E}_{\mathbb{P}}\left[e^{-\int_{t}^{T} r(u) d u} \xi \mid \mathcal{F}_{t}\right]
$$

The optimal portfolio-consumption strategy for the bid price is $\left(\pi^{*}, c^{*}\right)=\left(-\left(\sigma^{T}\right)^{-1} Y, \widehat{c}\right)$, and for the ask price is $\left(\pi^{*}, c^{*}\right)=\left(\left(\sigma^{T}\right)^{-1} Y, \widehat{c}\right)$, where $Y$ is given by (2.6).

Proof. We again only consider the case of the bid price, as the case of the ask price is similar. By the representation (2.10), we only need to solve

$$
\underset{(\pi, c) \in \Pi[t, T]}{\operatorname{ess.sup}} U_{t}\left(\xi+X_{T}^{0 ; \pi, c}\right) \text {. }
$$

For the initial wealth $X_{t}=0$, define the indirect utility $\bar{U}$ by subtracting the wealth $X^{0 ; \pi, c}$ from the original utility $U$ as

$$
\bar{U}_{s}=U_{s}\left(\xi+X_{T}^{0 ; \pi, c}\right)-X_{s}^{0 ; \pi, c} ; \quad \bar{Z}_{s}^{j}=Z_{s}^{j}-\sum_{i=1}^{n} \sigma_{i j}(s) \pi_{s}^{i}
$$

for $s \in[t, T]$. By (2.3) and (2.9), it is easy to verify that $(\bar{U}, \bar{Z})$ satisfies the following BSDE:

$$
\bar{U}_{t}=\xi+\int_{t}^{T}\left\{-r(u) \bar{U}_{u}+\left[v\left(u, c_{u}\right)-c_{u}\right]-\max _{\xi \in \Xi} \sum_{j=1}^{n} \xi_{u}^{j} Z_{u}^{j}\right\} d u-\int_{t}^{T} \bar{Z}_{u}^{T} d W_{u} .
$$

The maximum term in the above bracket can be rewritten in terms of $\bar{Z}$ as

$$
\max _{\xi \in \Xi} \sum_{j=1}^{n} \xi_{u}^{j} Z_{u}^{j}=\max _{\xi \in \Xi} \sum_{j=1}^{n} \xi_{u}^{j}\left(\bar{Z}_{u}^{j}+\sum_{i=1}^{n} \sigma_{i j}(u) \pi_{u}^{i}\right)=\delta_{\mathcal{O}}\left(\bar{Z}_{u},(\sigma(u))^{T} \pi_{u}\right),
$$

which is Lipschitz continuous in $\bar{Z}_{u}$, so the comparison principle holds for (2.19). For any $(\pi, c) \in \Pi$, we have

$$
v\left(t, c_{t}\right)-c_{t} \leq v^{*}(t) ; \quad-\delta_{O}\left(\bar{Z}_{t},(\sigma(t))^{T} \pi_{t}\right) \leq-d_{\mathcal{O}}\left(\bar{Z}_{t}, \mathcal{B}_{t}\right)
$$


and for $(\pi, c)=\left(\left(\sigma^{T}\right)^{-1} \operatorname{argmin}(\bar{Z}, \mathcal{B}), \widehat{c}\right)$, we have the equality:

$$
v\left(t, c_{t}^{*}\right)-c_{t}^{*}=v^{*}(t) ; \quad-\delta_{\mathcal{O}}\left(\bar{Z}_{t}, \operatorname{argmin}\left(\bar{Z}_{t}, \mathcal{B}_{t}\right)\right)=-d_{\mathcal{O}}\left(\bar{Z}_{t}, \mathcal{B}_{t}\right) .
$$

By the BSDE comparison principle, $\bar{U}_{t} \leq \bar{U}_{t}^{*}$ for any $(\pi, c) \in \Pi$, where $\bar{U}^{*}$ is the solution to BSDE:

$$
\bar{U}_{t}^{*}=\xi+\int_{t}^{T}\left(-r(u) \bar{U}_{u}^{*}+v^{*}(u)-d_{\mathcal{O}}\left(\bar{Z}_{u}^{*}, \mathcal{B}_{u}\right)\right) d u-\int_{t}^{T}\left(\bar{Z}_{u}^{*}\right)^{T} d W_{u},
$$

and $\left(\bar{U}_{t}, \bar{Z}_{t}\right)=\left(\bar{U}_{t}^{*}, \bar{Z}_{t}^{*}\right)$ for $(\pi, c)=\left(\left(\sigma^{T}\right)^{-1} \operatorname{argmin}(\bar{Z}, \mathcal{B}), \hat{c}\right)$.

Therefore,

$$
\operatorname{ess.sup}_{(\pi, c) \in \Pi[t, T]} U_{t}\left(\xi+X_{T}^{0 ; \pi, c}\right)=\operatorname{ess.sup}_{(\pi, c) \in \Pi[t, T]} \bar{U}_{t}=\bar{U}_{t}^{*}
$$

with the optimal portfolio-consumption strategy $\left(\pi^{*}, c^{*}\right)=\left(\left(\sigma^{T}\right)^{-1} \operatorname{argmin}\left(\bar{Z}^{*}, \mathcal{B}\right), \hat{c}\right)$. Finally, it is easy to verify that $\left(\bar{U}_{t}^{*}-\mathcal{R}(t), \bar{Z}_{t}^{*}\right)=\left(U_{t}^{b}, Z_{t}^{b}\right)$, which is the unique solution to BSDE (2.17).

Finally, if $\mathcal{A}=\mathbb{R}^{n}$, i.e. there is no trading constraint, $\mathcal{B}_{u}(\omega)$ is $\mathbb{R}^{n}$-valued as well, and

$$
d_{\mathcal{O}}\left(Z_{u}^{b}, \mathcal{B}_{u}(\omega)\right)=\min _{z \in \mathcal{B}_{u}(\omega)} \max _{\xi_{u}(\omega) \in \mathcal{O}}\left(\xi_{u}(\omega)\right)^{T}\left(Z_{u}^{b}+z\right)=0
$$

where $\operatorname{argmin}\left(Z_{u}^{b}, \mathcal{B}_{u}(\omega)\right)=-Z_{u}^{b}$. In this situation, the pricing BSDE (2.17) reduces to BSDE (2.6). By the uniqueness of the solution to (2.6), we have $\left(U^{b}, Z^{b}\right)=(D, Y)$. The optimal portfolio-consumption strategy in this situation reduces to

$$
\left(\pi^{*}, c^{*}\right)=\left(\left(\sigma^{T}\right)^{-1} \operatorname{argmin}\left(\bar{Z}^{*}, \mathcal{B}\right), \hat{c}\right)=\left(-\left(\sigma^{T}\right)^{-1} Y, \hat{c}\right) .
$$

In the following two sections, we will apply our BSDE representation results for utility indifference prices to European options and American options. Another potential application is to consider exotic options such as Parisian options. For example, Guo et al [12] consider the pricing problem of Parisian options also under the framework of Chen and Epstein [4. However, they use the idea of super-replication rather than utility indifference valuation, so they obtain pricing bounds rather than a price.

\section{Application to European Option}

In this section, we specify our model in a Markovian setting by assuming the payoff of the contingent claim having the form:

$$
\xi=\int_{t}^{T} \varrho(u) d u+\Psi\left(S_{T}\right)
$$

where $\varrho$ is the earning rate, and $\Psi$ is the final payoff of the contingent claim at the maturity $T$. They satisfy the following assumption:

Assumption 3.1 The earnings rate $\varrho(\cdot)$ is a continuous function, and the final payoff $\Psi$ is uniformly Lipschitz continuous:

$$
|\Psi(S)-\Psi(\bar{S})| \leq K|S-\bar{S}| \text { for } S, \bar{S} \in \mathbb{R}_{+}^{n},
$$

so $\Psi(\cdot)$ has linear growth. 
Under the above Markovian assumption, the bid price $P^{b}(t ; \xi)$ and the ask price $P^{s}(t ; \xi)$ can be written as functions of the time $t$ and the state $S_{t}: P^{b}\left(t, S_{t} ; \Psi\right) \triangleq P^{b}(t ; \xi)$, and $P^{s}\left(t, S_{t} ; \Psi\right) \triangleq P^{s}(t ; \xi)$. By Theorem 2.5. $P^{b}\left(t, S_{t} ; \Psi\right)$ and $P^{s}\left(t, S_{t} ; \Psi\right)$ are the solutions to the following BSDEs respectively:

$$
\begin{aligned}
& P^{b}\left(t, S_{t} ; \Psi\right)=\Psi\left(S_{T}\right)+\int_{t}^{T}\left[\varrho(u)-r(u) P^{b}\left(u, S_{u} ; \Psi\right)-d_{\mathcal{O}}\left(Z_{u}^{b}, \mathcal{B}_{u}\right)\right] d u-\int_{t}^{T}\left(Z_{u}^{b}\right)^{T} d W_{u} ; \\
& P^{s}\left(t, S_{t} ; \Psi\right)=\Psi\left(S_{T}\right)+\int_{t}^{T}\left[\varrho(u)-r(u) P^{s}\left(u, S_{u} ; \Psi\right)+d_{\mathcal{O}}\left(-Z_{u}^{s}, \mathcal{B}_{u}\right)\right] d u-\int_{t}^{T}\left(Z_{u}^{s}\right)^{T} d W_{u},
\end{aligned}
$$

where the state $S$ is given by (2.1). Moreover, by the nonlinear Feynman-Kac formula (see Theorem 4.2 of [10]), $P^{b}(t, S ; \Psi)$ and $P^{s}(t, S ; \Psi)$ are the unique viscosity solutions of the following semi-linear PDEs:

$$
\left\{\begin{array}{l}
-\partial_{t} P^{b}-\mathcal{L}_{0} P^{b}=\varrho(t)-d_{\mathcal{O}}\left((\sigma(t))^{T} S D_{S} P^{b}, \mathcal{B}_{t}\right) \text { in } \mathcal{N}_{T} ; \\
-\partial_{t} P^{s}-\mathcal{L}_{0} P^{s}=\varrho(t)+d_{\mathcal{O}}\left(-(\sigma(t))^{T} S D_{S} P^{s}, \mathcal{B}_{t}\right) \text { in } \mathcal{N}_{T} ; \\
P^{b}(T, S ; \Psi)=P^{s}(T, S ; \Psi)=\Psi(S), \quad S \in(0,+\infty)^{n},
\end{array}\right.
$$

where $\mathcal{N}_{T} \triangleq[0, T) \times(0,+\infty)^{n}$ and $S D_{S} P \triangleq\left(S_{1} \partial_{S_{1}} P, \cdots, S_{n} \partial_{S_{n}} P\right)^{T}$, and the operator $\mathcal{L}_{q}$ is given by

$$
\mathcal{L}_{q} \triangleq \sum_{i, j=1}^{n} \frac{1}{2} a_{i j}(t) S_{i} S_{j} \partial_{S_{i} S_{j}}+\sum_{i=1}^{n}\left[r(t)-q_{i}(t)\right] S_{i} \partial_{S_{i}}-r(t)
$$

with $a_{i j}(t) \triangleq \sum_{l=1}^{n} \sigma_{i l}(t) \sigma_{j l}(t)$. The term $q(\cdot)=\left(q_{1}(\cdot), \cdots, q_{n}(\cdot)\right)^{T}$ in the operator $\mathcal{L}_{q}$ is interpreted as the dividend rate of the risky assets $S$, and we shall see the bid price and the ask price under model uncertainty with trading constraint can be related to the risk-neutral price by adjusting the dividend rate of the underlying.

In order to investigate further properties of the bid and ask prices and their associated hedging strategies, we need to improve the regularities of $P^{b}$ and $P^{s}$. In the following, we present the strong solutions for PDEs (3.4).

Proposition 3.2 Suppose that Assumptions 2.1, 2.2 and 3.1 are satisfied. Then PDEs (3.4) have unique strong solutions with linear growth. Concretely speaking,

$$
P^{b}(t, S ; \Psi), P^{s}(t, S ; \Psi) \in W_{p, l o c}^{2,1}\left(\mathcal{N}_{T}\right) \cap C\left(\overline{\mathcal{N}}_{T}\right) \text { for any } p \geq 1
$$

and there exists a constant $C$ such that

$$
\left|P^{b}(t, S ; \Psi)\right|+\left|P^{s}(t, S ; \Psi)\right| \leq C(1+|S|) \text { for any }(t, S) \in \overline{\mathcal{N}}_{T}
$$

where $W_{p, \text { loc }}^{2,1}\left(\mathcal{N}_{T}\right)$ is the set of all functions whose restrictions on the domain $\mathcal{N}_{T}^{*}$ belong to $W_{p}^{2,1}\left(\mathcal{N}_{T}^{*}\right)$ for any compact subset $\mathcal{N}_{T}^{*}$ of $\mathcal{N}_{T}$, and $W_{p}^{2,1}\left(\mathcal{N}_{T}^{*}\right)$ is the completion of $C^{\infty}\left(\mathcal{N}_{T}^{*}\right)$ under the norm:

$$
\|P\|_{W_{p}^{2,1}\left(\mathcal{N}_{T}^{*}\right)} \triangleq\left[\int_{\mathcal{N}_{T}^{*}}\left(|P|^{p}+\left|\partial_{t} P\right|^{p}+\left|D_{S} P\right|^{p}+\left|D_{S}^{2} P\right|^{p}\right) d S d t\right]^{\frac{1}{p}}
$$

where $D_{S} P, D_{S}^{2} P$ denote the gradient and the Hessian matrix for $P$ with respect to $S$, respectively.

We shall show in the Appendix that PDEs (3.4) is a special case of the variational inequality (4.6) (see Theorem B.1). Hence, the above existence and regularity result is only a special case of the corresponding result for the variational inequality (4.6) in Proposition 4.5. 
In the rest of this section, we consider a concrete example of the priors set $\Theta$ by specifying the value set $\mathcal{O}$ of the corresponding kernel $\xi_{t}(\omega) \in \mathcal{O}$ :

$$
\mathcal{O}_{1} \triangleq\left\{x \in \mathbb{R}^{n}:-\underline{\kappa}_{i} \leq x_{i} \leq \bar{\kappa}_{i}, i=1, \cdots, n\right\}
$$

where $\underline{\kappa}_{i}, \bar{\kappa}_{i} \geq 0$. The corresponding priors set is denoted as $\Theta_{1}$, which is a generalization of the $\kappa$ ignorance model in Section 3.3 of [4] by taking $\underline{\kappa}_{i}=\bar{\kappa}_{i}$. With the above priors set $\Theta_{1}$, the support function $\delta_{\mathcal{O}_{1}}(\bar{z}, z)$ can be calculated as

$$
\delta_{\mathcal{O}_{1}}(\bar{z}, z) \triangleq \sum_{i=1}^{n}\left[\bar{\kappa}_{i}\left(\bar{z}_{i}+z_{i}\right)^{+}+\underline{\kappa}_{i}\left(\bar{z}_{i}+z_{i}\right)^{-}\right]=\bar{\kappa}^{T}(\bar{z}+z)^{+}+\underline{\kappa}^{T}(\bar{z}+z)^{-} .
$$

By Theorem 2.5] if there is no trading constraint, both the bid price and the ask price coincide with the risk-neutral price even with model uncertainty. In the following, we specify the admissible set $\Pi$ by restricting its values in $\mathcal{A}_{1}=[0, \infty)^{n}$, which is equivalent to short sale constraint. The corresponding admissible set is denoted as $\Pi_{1}$.

We denote the risk-neutral price under the Black-Scholes model with the dividend rate $q(\cdot)$ and the payoff $\Psi$ as $P^{0}(t, S ; q, \Psi)$, and the indifference prices with the priors set $\Theta_{1}$ and the admissible set $\Pi_{1}$ as $P^{1 m}(t, S ; \Psi)$ for $m \in\{b, s\}$. We can regard the Black-Scholes framework as a special case of our indifference pricing model by assuming $\delta_{\mathcal{O}_{0}}(\bar{z}, z)=0$.

Our main results in this section are the connections between the indifference prices and the risk-neutral prices with different dividend rates.

Proposition 3.3 Suppose that Assumptions 2.1, 2.2 and 3.1 are satisfied, the priors set is $\Theta_{1}$, and the admissible set is $\Pi_{1}$.

- If $\Psi(S)$ is increasing in each component $S_{i}$, then the bid price is given by

$$
P^{1 b}(t, S ; \Psi)=P^{0}(t, S ; \sigma \bar{\kappa}, \Psi)
$$

with the optimal portfolio-consumption strategy $\left(\pi^{*}, c^{*}\right)=(0, \hat{c})$, and the ask price is given by

$$
P^{1 s}(t, S ; \Psi)=P^{0}(t, S ; 0, \Psi)
$$

with the optimal portfolio-consumption strategy $\left(\pi^{*}, c^{*}\right)=\left(S D_{S} P^{0}, \hat{c}\right)$.

- If $\Psi(S)$ is decreasing in each component $S_{i}$, then the bid price is given by

$$
P^{1 b}(t, S ; \Psi)=P^{0}(t, S ; 0, \Psi)
$$

with the optimal portfolio-consumption strategy $\left(\pi^{*}, c^{*}\right)=\left(-S D_{S} P^{0}, \hat{c}\right)$, and the ask price is given by

$$
P^{1 s}(t, S ; \Psi)=P^{0}(t, S ; \sigma \bar{\kappa}, \Psi)
$$

with the optimal portfolio-consumption strategy $\left(\pi^{*}, c^{*}\right)=(0, \hat{c})$.

Proof. We only prove the case that $\Psi(S)$ is increasing in each component $S_{i}$, while the decreasing case is similar.

It is obvious from (2.1) that

$$
S_{T}^{i}=S_{t}^{i} \exp \left\{\int_{t}^{T}\left(r(u)-\frac{1}{2} \sum_{j=1}^{n}\left|\sigma_{i j}(u)\right|^{2}\right) d u+\int_{t}^{T} \sum_{j=1}^{n} \sigma_{i j}(u) d W_{u}^{j}\right\} .
$$


Hence, if $\Psi\left(S_{T}\right)$ is increasing in each component $S_{T}^{i}$, it is also increasing in $S_{t}^{i}$. By the BSDE comparison theorem, for $m \in\{b, s\}, P^{1 m}\left(t, S_{t} ; \Psi\right)$ is increasing in each component $S_{t}^{i}$ as well. Since $P^{1 m} \in W_{p, l o c}^{2,1}\left(\mathcal{N}_{T}\right)$ for any $p \geq 1$, the imbedding theorem for Sobolev space implies that $D_{S} P^{1 m} \in C\left(\mathcal{N}_{T}\right)$ if we choose $p>n+2$. Hence, $\partial_{S_{i}} P^{1 m} \geq 0$ for each $i=1,2, \cdots, n$. Recalling $\mathcal{B}_{t}=[0, \infty)^{n}$, we deduce that for the case of the bid price:

$$
\begin{aligned}
d_{\mathcal{O}_{1}}\left((\sigma(t))^{T} S D_{S} P^{1 b}, \mathcal{B}_{t}\right) & =\min _{z \in \mathcal{B}_{t}}\left\{\bar{\kappa}^{T}\left((\sigma(t))^{T} S D_{S} P^{1 b}+z\right)^{+}+\underline{\kappa}^{T}\left((\sigma(t))^{T} S D_{S} P^{1 b}+z\right)^{-}\right\} \\
& =(\sigma(t) \bar{\kappa})^{T} S D_{S} P^{1 b}
\end{aligned}
$$

with the optimizer $z^{*}=0$, or equivalently, $\pi^{*}=0$. For the case of the ask price:

$$
d_{\mathcal{O}_{1}}\left(-(\sigma(t))^{T} S D_{S} P^{1 s}, \mathcal{B}_{t}\right)=\min _{z \in \mathcal{B}_{t}}\left\{\bar{\kappa}^{T}\left(-(\sigma(t))^{T} S D_{S} P^{1 s}+z\right)^{+}+\underline{\kappa}^{T}\left(-(\sigma(t))^{T} S D_{S} P^{1 s}+z\right)^{-}\right\}=0
$$

with the optimizer $z^{*}=(\sigma(t))^{T} S D_{S} P^{1 s}$, or equivalently, $\pi^{*}=S D_{S} P^{1 s}$. Then the conclusions follow from the pricing equations (3.4).

Intuitively, if the payoff of an option is increasing with the prices of all the underlying stocks, an investor needs to hold a short position in each underlying stock in order to hedge a long position in this option, and a long position in each underlying stock in order to hedge a short position in this option. However, since there is short selling constraint (i.e. $\mathcal{A}_{1}=[0, \infty)^{n}$ ), hedging the long position of the option is impossible, and the best that the investor can do is not trading any underlying stocks. In turn, the investor has to compensate for the option price an equivalent dividend rate $\sigma \bar{\kappa}$.

The other observation is that the lower bound $\underline{\kappa}=\left(\underline{\kappa}_{1}, \cdots, \underline{\kappa}_{1}\right)^{T}$ in the priors set $\Theta_{1}$ does not impact the indifference prices $P^{1 b}$ and $P^{1 s}$, which is due to the asymmetric property of the trading constraint set $\mathcal{A}_{1}=[0, \infty)^{n}$. Moreover, the bid-ask spread $P^{1 b}-P^{1 s}$ is given in terms of the risk-neutral price with modified dividend rates: $\left|P^{0}(t, S ; 0, \Psi)-P^{0}(t, S ; \sigma \bar{\kappa}, \Psi)\right|$.

For general payoff $\Psi$, there are no explicit formulae for the bid price and the ask price. However, we can still have bounds on the indifference prices in terms of the risk-neutral price with modified dividend rates. Since PDEs (3.4) are a special case of the variational inequality (4.6) (see Theorem B.1), we will present the proof for the corresponding variational inequality (4.6) in Proposition4.7 and leave the following proof for PDEs (3.4) to the reader.

Proposition 3.4 Suppose that Assumptions 2.1, 2.2 and 3.1 are satisfied, the priors set is $\Theta_{1}$, and the admissible set is $\Pi_{1}$.

- The bid price satisfies the following inequality:

$$
\max \left\{P^{0}\left(t, S ; \sigma \bar{\kappa}, \underline{\Psi}^{+}\right), P^{0}\left(t, S ; 0, \underline{\Psi}^{-}\right)\right\} \leq P^{1 b}(t, S ; \Psi) \leq P^{0}(t, S ; q, \Psi)
$$

for any $q \in[0, \sigma \bar{\kappa}]$, and the optimal portfolio-consumption strategy is $\left(\pi^{*}, c^{*}\right)=\left(\left(S D_{S} P^{1 b}\right)^{-}, \hat{c}\right)$.

- The ask price satisfies the following inequality:

$$
P^{0}(t, S ; \sigma \bar{\kappa}, \Psi) \leq P^{1 s}(t, S ; \Psi) \leq \min \left\{P^{0}\left(t, S ; \sigma \bar{\kappa}, \bar{\Psi}^{-}\right), P^{0}\left(t, S ; 0, \bar{\Psi}^{+}\right)\right\},
$$

and the optimal portfolio-consumption strategy is $\left(\pi^{*}, c^{*}\right)=\left(\left(S D_{S} P^{1 s}\right)^{+}, \hat{c}\right)$.

Here $\underline{\Psi}^{+} / \underline{\Psi}^{-}$is any increasing/decreasing function bounded above by $\Psi$, and $\bar{\Psi}^{+} / \bar{\Psi}^{-}$is any increasing/decreasing function bounded below by $\Psi$. 
To finish this section, we investigate how the indifference prices converge to the corresponding riskneutral price when the priors set $\Theta_{1}$ shrinks to the probability set which only has negative densities, i.e. the positive part of the model uncertainty disappears.

Proposition 3.5 Suppose that Assumptions 2.1, 2.2 and 3.1 are satisfied, the priors set is $\Theta_{1}$, and the admissible set is $\Pi_{1}$. Then the bid price $P^{1 b}$ and the ask price $P^{1 s}$ converge to the risk-neutral price $P^{0}$ when the upper bound $\bar{\kappa}$ in the priors set $\Theta_{1}$ converges to zero. Concretely speaking,

$$
\left|P^{1 b}-P^{0}\right|+\left|P^{1 s}-P^{0}\right| \leq C \bar{\kappa}^{*}(1+|S|), \quad\left\|\left|P^{1 b}-P^{0}\right|+\left|P^{1 s}-P^{0}\right|\right\|_{W_{p}^{2,1}\left(\mathcal{N}_{T}^{*}\right)} \leq C_{\mathcal{N}_{T}^{*}} \bar{\kappa}^{*},
$$

where $\bar{\kappa}^{*}=\max \left\{\bar{\kappa}_{1}, \cdots, \bar{\kappa}_{n}\right\}$, and $\mathcal{N}_{T}^{*}$ is any compact subset of $\mathcal{N}_{T}$, and $C$ is a constant independent of $\mathcal{N}_{T}^{*}$, but $C_{\mathcal{N}_{T}^{*}}$ is a constant depending on $\mathcal{N}_{T}^{*}$.

We leave its proof in the Appendix.

\section{Application to American Option}

In this section, we extend our model to allow for an early exercise of the contingent claim. Assume that the contingent claim has the payoff:

$$
\xi=\int_{t}^{\tau \wedge T} \varrho(u) d u+\Gamma\left(\tau, S_{\tau}\right) \mathbf{1}_{\{\tau<T\}}+\Psi\left(S_{T}\right) I_{\{\tau=T\}},
$$

where $\tau \in \mathcal{U}[t, T]$ is any $\mathbb{F}$-stopping time valued in $[t, T], \varrho$ is the earning rate, and $\Gamma$ is the early payoff if the option is exercised before the maturity $T$, and $\Psi$ is the final payoff at the maturity $T$. The earning rate and the final payoff satisfy Assumption 3.1, and the early payoff $\Gamma$ satisfies the following assumption:

Assumption 4.1 The early payoff $\Gamma$ is uniformly Lipschitz continuous:

$$
|\Gamma(t, S)-\Gamma(\bar{t}, \bar{S})| \leq K(|t-\bar{t}|)+|S-\bar{S}|) \text { for } t, \bar{t} \in[0, T] \text { and } S, \bar{S} \in \mathbb{R}_{+}^{n},
$$

and is bounded above by $\Psi$.

Since the buyer of the American option has the right to exercise the option before the maturity $T$, the indifference price in Definition 2.3 needs to be modified accordingly. First, note that the investor's maximum utility satisfies the following time consistency property: For any $\mathbb{F}$-stopping time $\tau \in \mathcal{U}[t, T]$,

$$
\operatorname{ess.sup}_{(\pi, c) \in \Pi[t, T]} U_{t}\left(X_{T}^{X_{t} ; \pi, c}\right)=\operatorname{ess.sup}_{(\pi, c) \in \Pi[t, \tau]} U_{t}\left(\operatorname{ess.sup}_{(\pi, c) \in \Pi[\tau, T]} U_{\tau}\left(X_{T}^{X_{t} ; \pi, c}\right)\right)=\operatorname{ess.sup}_{(\pi, c) \in \Pi[t, \tau]} U_{t}\left(\mathcal{R}(\tau)+X_{\tau}^{X_{t} ; \pi, c}\right),
$$

where we used (2.15) in the last inequality. In other words, in order to have the time consistency property, the intermediate wealth at any $\mathbb{F}$-stopping time $\tau$ consists of not only the wealth $X_{\tau}^{X_{t} ; \pi, c}$, but also the value of the remaining optimal consumption $\mathcal{R}(\tau)$ from $\tau$ to the maturity $T$.

We modify Definition 2.3. and give the following definition of the indifference bid price of the American option.

Definition 4.2 The bid price $P^{b}\left(t, S_{t} ; \Gamma, \Psi\right)$ of the American option with the payoff $\int_{t}^{\tau \wedge T} \varrho(u) d u+$ $\mathbf{1}_{\{\tau<T\}} \Gamma\left(\tau, S_{\tau}\right)+\mathbf{1}_{\{\tau=T\}} \Psi\left(S_{T}\right)$, where $\tau \in \mathcal{U}[t, T]$ is the exercise time, is defined implicitly by the requirement that

$$
\begin{aligned}
\underset{(\pi, c) \in \Pi[t, T]}{\operatorname{ess.sup}} U_{t}\left(X_{T}^{X_{t} ; \pi, c}\right)= & \underset{\tau \in \mathcal{U}[t, T]}{\operatorname{ess} . \sup } \underset{(\pi, c) \in \Pi[t, \tau]}{\operatorname{ess.sup}} U_{t}\left(\int_{t}^{\tau \wedge T} \varrho(u) d u+\left(\mathcal{R}(\tau)+X_{\tau}^{X_{t}-P^{b}\left(t, S_{t} ; \Gamma, \Psi\right) ; \pi, c}\right.\right. \\
& \left.\left.+\Gamma\left(\tau, S_{\tau}\right)\right) \mathbf{1}_{\{\tau<T\}}+\left(X_{T}^{X_{t}-P^{b}\left(t, S_{t} ; \Gamma, \Psi\right) ; \pi, c}+\Psi\left(S_{T}\right)\right) \mathbf{1}_{\{\tau=T\}}\right) .(4.2)
\end{aligned}
$$


Similar to the proof of Theorem 2.4, it is easy to check that the optimization problem on RHS of (4.2) is translation invariant of its initial value:

$$
\begin{aligned}
& \underset{\tau \in \mathcal{U}[t, T](\pi, c) \in \Pi[t, \tau]}{\operatorname{ess.sup}} \operatorname{ess.sup} U_{t}\left(\int_{t}^{\tau \wedge T} \varrho(u) d u+\left(\mathcal{R}(\tau)+X_{\tau}^{0 ; \pi, c}+\Gamma\left(\tau, S_{\tau}\right)\right) \mathbf{1}_{\{\tau<T\}}\right. \\
& \left.+\left(X_{T}^{0 ; \pi, c}+\Psi\left(S_{T}\right)\right) \mathbf{1}_{\{\tau=T\}}\right)+X_{t}-P^{b}\left(t, S_{t} ; \Gamma, \Psi\right) .
\end{aligned}
$$

On the other hand, LHS of (4.2) is $\mathcal{R}(t)+X_{t}$ by (2.15). Therefore, the bid price of the American option is

$$
\begin{aligned}
P^{b}\left(t, S_{t} ; \Gamma, \Psi\right)= & \operatorname{ess.sup}_{\tau \in \mathcal{U}[t, T](\pi, c) \in \Pi[t, \tau]} \operatorname{ess.sup}_{t} U_{t}\left(\mathcal{R}(\tau)+X_{\tau}^{0 ; \pi, c}+\Gamma\left(\tau, S_{\tau}\right)\right) \mathbf{1}_{\{\tau<T\}} \\
& \left.+\left(X_{T}^{0 ; \pi, c}+\Psi\left(S_{T}\right)\right) \mathbf{1}_{\{\tau=T\}}+\int_{t}^{\tau \wedge T} \varrho(u) d u\right)-\mathcal{R}(t) .
\end{aligned}
$$

Theorem 4.3 Suppose that Assumptions 2.1, 2.2, 3.1 and 4.1 are satisfied. Then the bid price $P^{b}\left(t, S_{t} ; \Gamma, \Psi\right)=$ $U_{t}^{b}$, where $U_{t}^{b} \geq \Gamma\left(t, S_{t}\right)$ is the unique solution to reflected $B S D E$ :

$$
U_{t}^{b}=\Psi\left(S_{T}\right)+\int_{t}^{T}\left[\varrho(u)-r(u) U_{u}^{b}-d_{\mathcal{O}}\left(Z_{u}^{b}, \mathcal{B}_{u}\right)\right] d u+\int_{t}^{T} d K_{u}-\int_{t}^{T}\left(Z_{u}^{b}\right)^{T} d W_{u}
$$

for $\left(U^{b}, Z^{b}\right) \in L_{\mathbb{F}}^{2}(0, T ; \mathbb{R}) \times L_{\mathbb{F}}^{2}\left(0, T ; \mathbb{R}^{n}\right)$, and $K$ being continuous, increasing, starting from $K_{0}=0$, and satisfying the following Skorohod condition:

$$
\int_{0}^{T}\left[U_{u}^{b}-\Gamma\left(u, S_{u}\right)\right] d K_{u}=0
$$

The optimal portfolio-consumption strategy for the bid price is

$$
\left(\pi^{*}, c^{*}\right)=\left(\left(\sigma^{T}\right)^{-1} \operatorname{argmin}\left(Z^{b}, \mathcal{B}\right), \widehat{c}\right)
$$

and the optimal exercise time is

$$
\tau^{*}=\inf \left\{s \geq t: U_{s}^{b}=\Gamma\left(s, S_{s}\right)\right\} \wedge T
$$

Proof. Similar to the proof of Theorem of 2.5. define an indirect utility $\bar{U}$ by subtracting the wealth $X^{0 ; \pi, c}$ from the original utility $U$ as

$$
\begin{aligned}
& \bar{U}_{s}=U_{s}\left(\int_{t}^{\tau \wedge T} \varrho(u) d u+\left(\mathcal{R}(\tau)+X_{\tau}^{0 ; \pi, c}+\Gamma\left(\tau, S_{\tau}\right)\right) \mathbf{1}_{\{\tau<T\}}+\left(X_{T}^{0 ; \pi, c}+\Psi\left(S_{T}\right)\right) \mathbf{1}_{\{\tau=T\}}\right)-X_{s}^{0 ; \pi, c} ; \\
& \bar{Z}_{s}^{j}=Z_{s}^{j}-\sum_{i=1}^{n} \sigma_{i j}(s) \pi_{s}^{i}
\end{aligned}
$$

for $s \in[t, T]$. By (2.3) and (2.9), it is easy to verify that $(\bar{U}, \bar{Z})$ is the solution of the following BSDE:

$$
\begin{aligned}
\bar{U}_{t}= & \left(\mathcal{R}(\tau)+\Gamma\left(\tau, S_{\tau}\right)\right) \mathbf{1}_{\{\tau<T\}}+\Psi\left(S_{T}\right) \mathbf{1}_{\{\tau=T\}}-\int_{t}^{\tau \wedge T}\left(\bar{Z}_{u}\right)^{T} d W_{u} \\
& +\int_{t}^{\tau \wedge T}\left\{\varrho(u)-r(u) \bar{U}_{u}+\left[v\left(u, c_{u}\right)-c_{u}\right]-\delta_{\mathcal{O}}\left(\bar{Z}_{u},(\sigma(u))^{T} \pi_{u}\right)\right\} d u .
\end{aligned}
$$


By the BSDE comparison principle, $\bar{U}_{t} \leq \bar{U}_{t}^{*}$ for any $(\pi, c) \in \Pi[t, \tau]$, where $\bar{U}^{*}$ is the solution to BSDE:

$$
\begin{aligned}
\bar{U}_{t}^{*}= & \left(\mathcal{R}(\tau)+\Gamma\left(\tau, S_{\tau}\right)\right) \mathbf{1}_{\{\tau<T\}}+\Psi\left(S_{T}\right) \mathbf{1}_{\{\tau=T\}} \\
& +\int_{t}^{\tau \wedge T}\left\{\varrho(u)-r(u) \bar{U}_{u}^{*}+v^{*}(u)-d_{\mathcal{O}}\left(\bar{Z}_{u}^{*}, \mathcal{B}_{u}\right)\right\} d u-\int_{t}^{\tau \wedge T}\left(\bar{Z}_{u}^{*}\right)^{T} d W_{u} .
\end{aligned}
$$

and $\left(\bar{U}_{t}, \bar{Z}_{t}\right)=\left(\bar{U}_{t}^{*}, \bar{Z}_{t}^{*}\right)$ for $(\pi, c)=\left(\left(\sigma^{T}\right)^{-1} \operatorname{argmin}(\bar{Z}, \mathcal{B}), \hat{c}\right)$.

Furthermore, by Proposition 2.3 of El Karoui et al [9], $\bar{U}_{t}^{*} \leq \bar{U}_{t}^{* *}$ for any stopping time $\tau \in \mathcal{U}[t, T]$, where $\bar{U}_{t}^{* *} \geq \mathcal{R}(t)+\Gamma\left(t, S_{t}\right)$ is the solution of the following reflected BSDE:

$$
\bar{U}_{t}^{* *}=\Psi\left(S_{T}\right)+\int_{t}^{T}\left[\varrho(u)-r(u) \bar{U}_{u}^{* *}+v^{*}(u)-d_{\mathcal{O}}\left(\bar{Z}_{u}^{* *}, \mathcal{B}_{u}\right)\right] d u+\int_{t}^{T} d K_{u}-\int_{t}^{T}\left(\bar{Z}_{u}^{* *}\right)^{T} d W_{u},
$$

with the Skorohod condition:

$$
\int_{0}^{T}\left[\bar{U}_{u}^{* *}-\mathcal{R}(u)-\Gamma\left(u, S_{u}\right)\right] d K_{u}=0,
$$

and $\left(\bar{U}_{t}^{*}, \bar{Z}_{t}^{*}\right)=\left(\bar{U}_{t}^{* *}, \bar{Z}_{t}^{* *}\right)$ for $\tau=\tau^{*}$. Therefore,

$$
\begin{aligned}
\operatorname{ess.sup}_{\tau \in \mathcal{U}[t, T]} \operatorname{ess.sup}(\pi, c) \in \Pi[t, \tau] & U_{t}\left(\int_{t}^{\tau \wedge T} \varrho(u) d u+\left(\mathcal{R}(\tau)+X_{\tau}^{0 ; \pi, c}+\Gamma\left(\tau, S_{\tau}\right)\right) \mathbf{1}_{\{\tau<T\}}\right. \\
& \left.+\left(X_{T}^{0 ; \pi, c}+\Psi\left(S_{T}\right)\right) \mathbf{1}_{\{\tau=T\}}\right) \\
= & \operatorname{ess.sup}_{\tau \in \mathcal{U}[t, T]} \operatorname{ess.sup}_{(\pi, c) \in \Pi[t, \tau]} \bar{U}_{t}=\underset{\tau \in \mathcal{U}[t, T]}{\operatorname{ess.sup}} \bar{U}_{t}^{*}=\bar{U}_{t}^{* *}
\end{aligned}
$$

with the optimal portfolio-consumption strategy $\left(\pi^{*}, c^{*}\right)=\left(\left(\sigma^{T}\right)^{-1} \operatorname{argmin}\left(\bar{Z}^{* *}, \mathcal{B}\right), \hat{c}\right)$ and the optimal exercise time $\tau^{*}$.

Finally, it is easy to verify that $\left(\bar{U}_{t}^{* *}-\mathcal{R}(t), \bar{Z}_{t}^{* *}\right)=\left(U_{t}^{b}, Z_{t}^{b}\right)$, which is the unique solution to the reflected BSDE (4.3).

It is important to recall that it is the buyer of the claim who decides when the contract is exercised. The writer of the derivative does not have this opportunity and, therefore, she will have to maximize his utility contingently on the buyer's optimal actions. In a sense, the valuation problem of the writer reduces to a barrier type with expiration given by the buyer's optimally chosen exercise time $\tau^{*}$, and the payoff is $\mathbf{1}_{\left\{\tau^{*}<T\right\}} \Gamma\left(\tau, S_{\tau}\right)+\mathbf{1}_{\left\{\tau^{*}=T\right\}} \Psi\left(S_{T}\right)$. This asymmetry is not observed in complete markets where there is a unique price. However, in incomplete markets such asymmetries naturally emerge and give rise to realistic price spreads.

Proposition 4.4 The ask price $P^{s}\left(t, S_{t} ; \Gamma, \Psi\right)$ of the American option with the payoff $\int_{t}^{\tau^{*} \wedge T} \varrho(u) d u+$ $\mathbf{1}_{\left\{\tau^{*}<T\right\}} \Gamma\left(\tau^{*}, S_{\tau^{*}}\right)+\mathbf{1}_{\left\{\tau^{*}=T\right\}} \Psi\left(S_{T}\right)$ is defined implicitly by the requirement that

$$
\begin{aligned}
\operatorname{ess.sup}_{(\pi, c) \in \Pi[t, T]} U_{t}\left(X_{T}^{X_{t} ; \pi, c}\right)= & \operatorname{ess.sup}_{(\pi, c) \in \Pi\left[t, \tau^{*}\right]} U_{t}\left(\int_{t}^{\tau^{*} \wedge T} \varrho(u) d u+\left(\mathcal{R}\left(\tau^{*}\right)+X_{\tau^{*}}^{X_{t}-P^{b}\left(t, S_{t} ; \Gamma, \Psi\right) ; \pi, c}\right.\right. \\
& \left.\left.+\Gamma\left(\tau^{*}, S_{\tau^{*}}\right)\right) \mathbf{1}_{\left\{\tau^{*}<T\right\}}+\left(X_{T}^{X_{t}-P^{b}\left(t, S_{t} ; \Gamma, \Psi\right) ; \pi, c}+\Psi\left(S_{T}\right)\right) \mathbf{1}_{\left\{\tau^{*}=T\right\}}\right) .
\end{aligned}
$$

Suppose that Assumptions [2.1, 2.2, 3.1 and 4.1 are satisfied. Then the ask price $P^{s}\left(t, S_{t} ; \Gamma, \Psi\right)=U_{t}^{s}$, where $U^{s}$ is the unique solution to BSDE:

$$
\begin{aligned}
U_{t}^{s}= & \Gamma\left(\tau^{*}, S_{\tau^{*}}\right) \mathbf{1}_{\left\{\tau^{*}<T\right\}}+\Psi\left(S_{T}\right) \mathbf{1}_{\left\{\tau^{*}=T\right\}} \\
& +\int_{t}^{\tau^{*} \wedge T}\left[\varrho(u)-r(u) U_{u}^{s}+d_{\mathcal{O}}\left(-Z_{u}^{s}, \mathcal{B}_{u}\right)\right] d u-\int_{t}^{\tau^{*} \wedge T}\left(Z_{u}^{s}\right)^{T} d W_{u}
\end{aligned}
$$


for $\left(U^{s}, Z^{s}\right) \in L_{\mathbb{F}}^{2}(0, T ; \mathbb{R}) \times L_{\mathbb{F}}^{2}\left(0, T ; \mathbb{R}^{n}\right)$. The optimal portfolio-consumption strategy for the ask price is

$$
\left(\pi^{*}, c^{*}\right)=\left(\left(\sigma^{T}\right)^{-1} \operatorname{argmin}\left(-Z^{s}, \mathcal{B}\right), \widehat{c}\right) .
$$

Proof. The proof is similar to the proof of Theorem 2.5, so we omit it.

By the nonlinear Feynman-Kac formula, $P^{b}(t, S ; \Gamma, \Psi)$ and $P^{s}(t, S ; \Gamma, \Psi)$ are the unique viscosity solutions of the following variational inequality and semi-linear PDE respectively:

$$
\left\{\begin{array}{l}
-\partial_{t} P^{b}-\mathcal{L}_{0} P^{b}=\varrho(t)-d_{\mathcal{O}}\left((\sigma(t))^{T} S D_{S} P^{b}, \mathcal{B}_{t}\right) \text { if } P^{b}>\Gamma \text { and }(t, S) \in \mathcal{N}_{T} \\
-\partial_{t} P^{b}-\mathcal{L}_{0} P^{b} \geq \varrho(t)-d_{\mathcal{O}}\left((\sigma(t))^{T} S D_{S} P^{b}, \mathcal{B}_{t}\right) \text { if } P^{b}=\Gamma \text { and }(t, S) \in \mathcal{N}_{T} \\
P^{b}(T, S ; \Gamma, \Psi)=\Psi(S),
\end{array}\right.
$$

and

$$
\left\{\begin{array}{l}
-\partial_{t} P^{s}-\mathcal{L}_{0} P^{s}=\varrho(t)+d_{\mathcal{O}}\left(-(\sigma(t))^{T} S D_{S} P^{s}, \mathcal{B}_{t}\right) \text { in }\left\{P^{b}>\Gamma\right\} ; \\
P^{s}=\Gamma \text { in }\left\{P^{b}=\Gamma\right\} ; \quad P^{s}(T, S ; \Gamma, \Psi)=\Psi(S) .
\end{array}\right.
$$

We have the following existence and regularity results for the strong solutions of (4.6) and (4.7). Note that the early payoff function $\Gamma$ usually takes the form $\Gamma=\max \left\{\Gamma_{1}, 0\right\}$, where $\Gamma_{1}$ is some payoff if the investor exercises the option. For example, the early payoff function of American call/put option is just $(S-K)^{+} /(K-S)^{+}$with the strike price $K$.

Proposition 4.5 Suppose that Assumptions 2.1, 2.2, and 3.1 are satisfied. Moreover, the early payoff function $\Gamma$ has the form $\Gamma=\max \left\{\Gamma_{1}, \Gamma_{2}\right\}$, where both $\Gamma_{1}$ and $\Gamma_{2}$ satisfy Assumptions 4.1. with $D_{S}^{2} \Gamma_{1}$ and $D_{S}^{2} \Gamma_{2}$ having polynomial growth, i.e., there exist a positive constant $C$ and a positive integer $N$ such that

$$
\left|D_{S}^{2} \Gamma_{1}\right|+\left|D_{S}^{2} \Gamma_{2}\right| \leq C\left(1+|S|^{N}\right) .
$$

Then both (4.6) and (4.7) have unique strong solutions with linear growth. Concretely speaking,

$$
\begin{aligned}
& P^{b}(t, S ; \Gamma, \Psi) \in W_{p, l o c}^{2,1}\left(\mathcal{N}_{T}\right) \cap C\left(\overline{\mathcal{N}}_{T}\right) ; \\
& P^{s}(t, S ; \Gamma, \Psi) \in W_{p, l o c}^{2,1}\left(\mathcal{N}_{T} \cap\left\{P^{b} \geq \Gamma\right\}\right) \cap C\left(\overline{\mathcal{N}}_{T}\right),
\end{aligned}
$$

and there exists a constant $C$ such that

$$
\left|P^{b}(t, S ; \Gamma, \Psi)\right|+\left|P^{s}(t, S ; \Gamma, \Psi)\right| \leq C(1+|S|) .
$$

We leave its proof in the Appendix.

In the rest of this section, we consider a concrete example with the priors set $\Theta_{1}$ and the admissible set $\Pi_{1}$, where $\Theta_{1}$ and $\Pi_{1}$ are given in the last section. We denote the risk-neutral American option price with the dividend rate $q(\cdot)$ and the payoff $\int_{t}^{\tau \wedge T} \varrho(u) d u+\mathbf{1}_{\{\tau<T\}} \Gamma\left(\tau, S_{\tau}\right)+\mathbf{1}_{\{\tau=T\}} \Psi\left(S_{T}\right)$ as $P^{0}(t, S ; q, \Gamma, \Psi)$, and the indifference prices with the priors set $\Theta_{1}$ and the admissible set $\Pi_{1}$ as $P^{1 m}(t, S ; \Gamma, \Psi)$ for $m \in\{b, s\}$. We can regard the standard American option pricing framework as a special case of our indifference pricing model for the bid price by assuming $\delta_{\mathcal{O}_{0}}(\bar{z}, z)=0$.

Proposition 4.6 Suppose that the assumptions in Proposition 4.5 are satisfied, the priors set is $\Theta_{1}$, and the admissible set is $\Pi_{1}$. 
- If both $\Gamma(S)$ and $\Psi(S)$ are increasing in each component $S_{i}$, then the bid price is given by

$$
P^{1 b}(t, S ; \Gamma, \Psi)=P^{0}(t, S ; \sigma \bar{\kappa}, \Gamma, \Psi)
$$

with the optimal portfolio-consumption strategy $\left(\pi^{*}, c^{*}\right)=(0, \hat{c})$, and the optimal exercise time

$$
\tau^{*}=\widehat{\tau} \triangleq \inf \left\{s \geq t: P^{1 b}\left(s, S_{s} ; \Gamma, \Psi\right)=\Gamma\left(s, S_{s}\right)\right\} \wedge T .
$$

The ask price is bounded by

$$
P^{1 b}(t, S ; \Gamma, \Psi) \leq P^{1 s}(t, S ; \Gamma, \Psi) \leq P^{0}(t, S ; 0, \Gamma, \Psi)
$$

with the optimal portfolio-consumption strategy $\left(\pi^{*}, c^{*}\right)=\left(S D_{S} P^{1 s}, \hat{c}\right)$.

- If both $\Gamma(S)$ and $\Psi(S)$ are decreasing in each component $S_{i}$, then the bid price is given by

$$
P^{1 b}(t, S ; \Gamma, \Psi)=P^{0}(t, S ; 0, \Gamma, \Psi)
$$

with the optimal portfolio-consumption strategy $\left(\pi^{*}, c^{*}\right)=\left(-S D_{S} P^{0}, \hat{c}\right)$, and the optimal exercise time $\tau^{*}=\widehat{\tau}$. The ask price is bounded by

$$
P^{1 b}(t, S ; \Gamma, \Psi) \leq P^{1 s}(t, S ; \Gamma, \Psi) \leq P^{0}(t, S ; \sigma \bar{\kappa}, \Gamma, \Psi)
$$

with the optimal portfolio-consumption strategy $\left(\pi^{*}, c^{*}\right)=(0, \hat{c})$.

Proof. We only prove the case that $\Psi(S)$ is increasing in each component $S_{i}$, while the decreasing case is similar. Let $P^{0}, P^{1 s}$ and $P^{1 b}$ denote $P^{0}(t, S ; 0, \Gamma, \Psi), P^{1 s}(t, S ; \Gamma, \Psi)$ and $P^{1 b}(t, S ; \Gamma, \Psi)$, respectively.

First, as in the proof of Proposition 3.3. by the BSDE comparison theorem and the regularity of the strong solution, we deduce that $D_{S_{i}} P^{1 m} \geq 0$ for $m \in\{b, s\}$. Therefore, for the case of the bid price,

$$
d_{\mathcal{O}_{1}}\left((\sigma(t))^{T} S D_{S} P^{1 b}, \mathcal{B}_{t}\right)=(\sigma(t) \bar{\kappa})^{T} S D_{S} P^{1 b}
$$

with the optimizer $\pi^{*}=0$, and for the case of the ask price:

$$
d_{\mathcal{O}_{1}}\left(-(\sigma(t))^{T} S D_{S} P^{1 s}, \mathcal{B}_{t}\right)=0
$$

with the optimizer $\pi^{*}=S D_{S} P^{1 s}$. Then $P^{1 b}(t, S ; \Gamma, \Psi)=P^{0}(t, S ; \sigma \bar{\kappa}, \Gamma, \Psi)$ follows from the pricing equation (4.6).

Different from the European option case, the optimal trading strategy of the American option's holder affects the seller's ask price. In other words, the solution of (4.6) affects the solution of (4.7), so we can not expect that the ask price is equal to the price in the standard Black-Scholes market.

We first prove the lower bound of the ask price. It is clear that $P^{1 s}=P^{1 b}=\Gamma$ in the domain $\left\{P^{1 b}=\Gamma\right\}$ from (4.7). In the domain $\left\{P^{1 b}>\Gamma\right\}$, note that $d_{\mathcal{O}_{1}}\left(\cdot, \mathcal{B}_{u}\right) \geq 0$, then we have

$$
\begin{aligned}
-\partial_{t} P^{1 b}-\mathcal{L}_{0} P^{1 b} & =\varrho(t)-d_{\mathcal{O}_{1}}\left((\sigma(t))^{T} S D_{S} P^{1 b}, \mathcal{B}_{u}\right) \\
& \leq \varrho(t)+d_{\mathcal{O}_{1}}\left(-(\sigma(t))^{T} S D_{S} P^{1 s}, \mathcal{B}_{u}\right)=-\partial_{t} P^{1 s}-\mathcal{L}_{0} P^{1 s} .
\end{aligned}
$$

The continuity and the terminal and boundary conditions of $P^{1 b}, P^{1 s}$ imply $P^{1 b}=P^{1 s}$ on the parabolic boundary of $\left\{P^{1 b}>\Gamma\right\}$. By applying Lemma A.6, we deduce $P^{1 b} \leq P^{1 s}$.

Next, we use the similar method to prove the upper bound of the ask price. Since $P^{0} \geq \Gamma$, we derive that $P^{0} \geq P^{1 s}$ in the domain $\left\{P^{1 b}=\Gamma\right\}$ from (4.7). Note that $d_{\mathcal{O}_{0}}\left(\cdot, \mathcal{B}_{u}\right)=0=d_{\mathcal{O}_{1}}\left(-(\sigma(t))^{T} S D_{S} P^{1 s}, \mathcal{B}_{t}\right)$, we deduce that in the domain $\left\{P^{1 b}>\Gamma\right\}$

$$
-\partial_{t} P^{1 s}-\mathcal{L}_{0} P^{1 s}=\varrho(t) \leq-\partial_{t} P^{0}-\mathcal{L}_{0} P^{0} .
$$

Moreover, the continuity of $P^{1 s}$ implies that $P^{1 s}=\Gamma \leq P^{0}$ on $\partial_{p}\left\{P^{1 b}>\Gamma\right\} \cap \mathcal{N}_{T}$. On the other hand, $P^{1 s}=P^{0}$ on $\partial_{p}\left\{P^{1 b}>\Gamma\right\} \cap \partial_{p} \mathcal{N}_{T}$. By applying Lemma A.6, we deduce $P^{1 s} \leq P^{0}$. 
Proposition 4.7 Suppose that the assumptions in Proposition 4.5 are satisfied, the priors set is $\Theta_{1}$, and the admissible set is $\Pi_{1}$. Then we have

- The bid price satisfies the following inequality:

$$
\begin{aligned}
& \max \left\{P^{0}\left(t, S ; \sigma \bar{\kappa}, \underline{\Gamma}^{+}, \underline{\Psi}^{+}\right), P^{0}\left(t, S ; 0, \underline{\Gamma}^{-}, \underline{\Psi}^{-}\right)\right\} \\
\leq & P^{1 b}(t, S ; \Gamma, \Psi) \leq P^{0}(t, S ; q, \Gamma, \Psi), \forall q \in[0, \sigma \bar{\kappa}] .
\end{aligned}
$$

The optimal portfolio-consumption strategy is $\left(\pi^{*}, c^{*}\right)=\left(\left(S D_{S} P^{1 b}\right)^{-}, \hat{c}\right)$, and the optimal exercise time $\tau^{*}=\widehat{\tau}$.

- The ask price satisfies the following inequality:

$$
\begin{aligned}
& P^{1 b}(t, S ; \Gamma, \Psi) \leq P^{1 s}(t, S ; \Gamma, \Psi) \\
\leq & \min \left\{P^{0}\left(t, S ; \sigma \bar{\kappa}, \bar{\Gamma}^{-}, \bar{\Psi}^{-}\right), P^{0}\left(t, S ; 0, \bar{\Gamma}^{+}, \bar{\Psi}^{+}\right)\right\} .
\end{aligned}
$$

The optimal portfolio-consumption strategy is $\left(\pi^{*}, c^{*}\right)=\left(\left(S D_{S} P^{1 s}\right)^{+}, \hat{c}\right)$,

where $\underline{\Gamma}^{+} / \underline{\Gamma}^{-}$is any increasing/decreasing function bounded above by $\Gamma$, and $\bar{\Gamma}^{+} / \bar{\Gamma}^{-}$is any increasing/decreasing function bounded below by $\Gamma$.

Proof. We only prove the case of the bid price, as the case of the ask price is similar. Let $P^{0}$ and $P^{1 b}$ denote $P^{0}(t, S ; q, \Gamma, \Psi)$ and $P^{1 b}(t, S ; \Gamma, \Psi)$, respectively.

Note that if the final payoff $\Psi$ does not have any monotone property, the sign of $D_{S} P^{1 b}$ is indefinite. In this case,

$$
\begin{aligned}
& d_{\mathcal{O}_{1}}\left((\sigma(t))^{T} S D_{S} P^{1 b}, \mathcal{B}_{t}\right) \\
= & \min _{z \in \mathcal{B}_{t}}\left\{\bar{\kappa}^{T}\left((\sigma(t))^{T} S D_{S} P^{1 b}+z\right)^{+}+\underline{\kappa}^{T}\left((\sigma(t))^{T} S D_{S} P^{1 b}+z\right)^{-}\right\} \\
= & (\sigma(t) \bar{\kappa})^{T}\left(S D_{S} P^{1 b}\right)^{+}
\end{aligned}
$$

with the optimizer $z^{*}=(\sigma(t))^{T}\left(S D_{S} P^{1 b}\right)^{-}$, or equivalently, $\pi^{*}=\left(S D_{S} P^{1 b}\right)^{-}$. Denote

$$
\widetilde{\varrho}(t) \triangleq\left(-\partial_{t} P^{1 b}-\mathcal{L}_{q} P^{1 b}\right)-\left(-\partial_{t} P^{1 b}-\mathcal{L}_{0} P^{1 b}\right)+\varrho(t)-d_{\mathcal{O}_{1}}\left((\sigma(t))^{T} S D_{S} P^{1 b}, \mathcal{B}_{t}\right) .
$$

Then we can rewrite the variational inequality (4.6) as follows

$$
\left\{\begin{array}{l}
-\partial_{t} P^{1 b}-\mathcal{L}_{q} P^{1 b}=\widetilde{\varrho}(t) \text { if } P^{1 b}>\Gamma \text { and }(t, S) \in \mathcal{N}_{T} \\
-\partial_{t} P^{1 b}-\mathcal{L}_{q} P^{1 b} \geq \widetilde{\varrho}(t) \text { if } P^{1 b}=\Gamma \text { and }(t, S) \in \mathcal{N}_{T} \\
P^{1 b}(T, S)=\Psi(S) .
\end{array}\right.
$$

On the other hand, $P^{0}$ satisfies

$$
\left\{\begin{array}{l}
-\partial_{t} P^{0}-\mathcal{L}_{q} P^{0}=\varrho(t) \text { if } P^{0}>\Gamma \text { and }(t, S) \in \mathcal{N}_{T} \\
-\partial_{t} P^{0}-\mathcal{L}_{q} P^{0} \geq \varrho(t) \text { if } P^{0}=\Gamma \text { and }(t, S) \in \mathcal{N}_{T} \\
P^{0}(T, S)=\Psi(S)
\end{array}\right.
$$

Moreover, it is not difficult to check that

$$
\widetilde{\varrho}(t)=-\left[(\sigma(t) \bar{\kappa})^{T}-q(t)^{T}\right]\left(S D_{S} P^{1 b}\right)^{+}-q(t)^{T}\left[\left(S D_{S} P^{1 b}\right)^{+}-S D_{S} P^{1 b}\right]+\varrho(t) \leq \varrho(t) .
$$


By applying Lemma A.6 wave the upper bound of the bid price:

$$
P^{1 b}\left(t, S_{t} ; \Gamma, \Psi\right) \leq P^{0}\left(t, S_{t} ; q, \Gamma, \Psi\right), \quad \forall q \in[0, \sigma \bar{\kappa}] .
$$

On the other hand, $P^{1 b}(t, S ; \Gamma, \Psi)$ and $P^{1 b}\left(t, S_{t} ; \underline{\Gamma}^{+} / \underline{\Gamma}^{-}, \underline{\Psi}^{+} / \underline{\Psi}^{-}\right)$satisfy the same differential equation, but with different terminal values. Lemma A.6 implies that $P^{1 b}(t, S ; \Gamma, \Psi)$ is larger since it has larger terminal value and obstacle. By Proposition 4.6, the bid price associated with the payoff $\underline{\Gamma}^{+}, \underline{\Psi}^{+}$is $P^{0}\left(t, S_{t} ; \sigma \bar{\kappa}, \underline{\Gamma}^{+}, \underline{\Psi}^{+}\right)$, and the bid price associated with the payoff $\underline{\Gamma}^{-}, \underline{\Psi}^{-}$is $P^{0}\left(t, S_{t} ; 0, \underline{\Gamma}^{-}, \underline{\Psi}^{-}\right)$. Hence, we have the lower bound of the bid price:

$$
P^{1 b}(t, S ; \Gamma, \Psi) \geq \max \left\{P^{0}\left(t, S_{t} ; \sigma \bar{\kappa}, \underline{\Gamma}^{+}, \underline{\Psi}^{+}\right), P^{0}\left(t, S_{t} ; 0, \underline{\Gamma}^{-}, \underline{\Psi}^{-}\right)\right\} .
$$

To finish this section, we present a converge result of the indifference price to the risk-neutral price for the American option as in Proposition 3.5 for the European option case.

Proposition 4.8 Suppose the assumptions in Proposition 4.5 are satisfied, the priors set is $\Theta_{1}$, and the admissible set is $\Pi_{1}$. Then the bid price $P^{1 b}$ and the ask price $P^{1 s}$ converge to the risk-neutral price $P^{0}$ when the upper bound $\bar{\kappa}$ in the priors set $\Theta_{1}$ converges to zero, Concretely speaking,

$$
\left|P^{1 b}-P^{0}\right|+\left|P^{1 s}-P^{0}\right| \leq C \bar{\kappa}^{*}(1+|S|) \text { in } \mathcal{N}_{T},
$$

where $C$ is a constant independent of $\bar{\kappa}^{*}$.

We leave its proof in the Appendix.

\section{Appendix}

\section{A Some Results of Relevant PDEs}

In this Appendix, we provide some technical details on the results of relevant pricing PDEs for the utility indifference prices. The main references are Lieberman 21] and Ladyzenskaja et al 20].

We consider the following semi-linear parabolic PDE in a general form:

$$
\begin{cases}-\partial_{t} P-L P=0 & \text { in } \mathcal{Q}_{T} \\ P=\Psi & \text { on } \partial_{p} \mathcal{Q}_{T}\end{cases}
$$

where $\partial_{p} \mathcal{Q}_{T}$ is the backward parabolic boundary of $\mathcal{Q}_{T}$, which is a bounded or unbounded backward parabolic domain, and the differential operator

$$
L P \triangleq \sum_{i, j=1}^{n} a_{i j} \partial_{S_{i} S_{j}} P+\sum_{i=1}^{n} b_{i} \partial_{S_{i}} P+c P+F\left(t, S, P, D_{S} P\right) .
$$

Assumption A.1 The coefficient function a is continuous in $\overline{\mathcal{Q}}_{T}$, and there exists a positive constant $K$ such that

$$
|a(t, S)|(1+|S|)^{-2}+|b(t, S)|(1+|S|)^{-1}+|c(t, S)| \leq K \text { for any }(t, S) \in \mathcal{Q}_{T} .
$$

Assumption A.2 There exists a positive constant $K$ such that

$$
\left|F\left(t, S, u_{1}, v_{1}\right)-F\left(t, S, u_{2}, v_{1}\right)\right| \leq K(1+|S|)\left(\left|u_{1}-u_{2}\right|+\left|v_{1}-v_{2}\right|\right)
$$

for any $(t, S) \in \mathcal{Q}_{T}, u_{1}, u_{2} \in \mathbb{R}, v_{1}, v_{2} \in \mathbb{R}^{n}$. 
First, we present the existence and uniqueness result for the strong solution of (A.1):

Lemma A.3 Let $\mathcal{Q}_{T}=[0, T) \times \mathcal{Q}$ where $\mathcal{Q}$ is a bounded open domain in $\mathbb{R}^{n}$ with $C^{2}$ boundary. Suppose that Assumptions A.1 and A.2 are satisfied, and a satisfies the uniformly positive definite condition in $\mathcal{Q}_{T}$, i.e.,

$$
\sum_{i, j=1}^{n} a_{i j}(t, S) \xi_{i} \xi_{j} \geq|\xi|^{2} / K \text { for any }(t, S) \in \mathcal{Q}_{T}, \xi \in \mathbb{R}^{n} .
$$

Moreover, $F(\cdot, \cdot, 0,0) \in L^{p}\left(\mathcal{Q}_{T}\right), \Psi \in W_{p}^{2,1}\left(\mathcal{Q}_{T}\right)$ with some $p \geq 1$.

Then (A.1) has a unique strong solution $P \in W_{p}^{2,1}\left(\mathcal{Q}_{T}\right)$. Moreover, the following estimate holds

$$
\|P\|_{W_{p}^{2,1}\left(\mathcal{Q}_{T}\right)} \leq C\left(\|F(\cdot, \cdot, 0,0)\|_{L^{p}\left(\mathcal{Q}_{T}\right)}+\|\Psi\|_{W_{p}^{2,1}\left(\mathcal{Q}_{T}\right)}\right),
$$

where the constant $C$ depends on $K, p, n, \mathcal{Q}_{T}$.

Next, we present the interior $W_{p}^{2,1}$ estimate and $C^{\alpha}$ estimate with local boundary for PDE (A.1), which is the key tool to study the problem with low regularity on the boundary, or the problem in unbounded domain.

Lemma A.4 Let $\mathcal{Q}_{T}$ be a bounded or unbounded backward parabolic domain, and $\mathcal{Q}_{T}^{*}$ be a compact subset of $\mathcal{Q}_{T}$. Suppose that Assumptions $A .1$ and A.2 are satisfied, and a satisfies the uniformly positive definite condition in $\mathcal{Q}_{T}^{*}$. Moreover, $F(\cdot, \cdot, 0,0) \in L^{p}\left(\mathcal{Q}_{T}^{*}\right)$ with some $p \geq 1$.

Then the following estimate holds

$$
\|P\|_{W_{p}^{2,1}\left(\mathcal{Q}_{T}^{* *}\right)} \leq C\left(\|F(\cdot, \cdot, 0,0)\|_{L^{p}\left(\mathcal{Q}_{T}^{*}\right)}+\|P\|_{L^{p}\left(\mathcal{Q}_{T}^{*}\right)}\right),
$$

where $\mathcal{Q}_{T}^{* *}$ is a compact subset of $\mathcal{Q}_{T}^{*}$, and $C$ depends on $K, p, n, \mathcal{Q}_{T}^{* *}$, and $\operatorname{dist}\left(\mathcal{Q}_{T}, \mathcal{Q}_{T}^{*}\right), \operatorname{dist}\left(\mathcal{Q}_{T}^{*}, \mathcal{Q}_{T}^{* *}\right)$.

Lemma A.5 Let $\mathcal{Q}_{T} \triangleq[0, T) \times \mathcal{Q}$ where $\mathcal{Q}$ is an open domain in $\mathbb{R}^{n}$ with continuous boundary, and $\mathcal{Q}^{*}$ be a compact subset of $\mathcal{Q}$. Suppose that Assumptions A.1 and A.2 are satisfied, and a is uniformly positive definite in $\mathcal{Q}_{T}^{*} \triangleq[0, T) \times \mathcal{Q}^{*}$. Moreover, $F(\cdot, \cdot, 0,0) \in L^{p}\left(\mathcal{Q}_{T}^{*}\right), \Psi \in C^{\alpha, \alpha / 2}\left(\overline{\mathcal{Q}^{*}}\right)$ with some $p>n+2, \alpha \in(0,1)$.

Then there exists a constant $\beta \in(0, \alpha)$ such that the following estimate holds

$$
\|P\|_{C^{\beta, \beta / 2}\left(\overline{\mathcal{Q}_{T}^{* *}}\right)} \leq C\left(\|F(\cdot, \cdot, 0,0)\|_{L^{p}\left(\mathcal{Q}_{T}^{*}\right)}+\|P\|_{L^{\infty}\left(\mathcal{Q}_{T}^{*}\right)}+\|\Psi\|_{C^{\alpha, \alpha / 2}\left(\overline{\mathcal{Q}^{*}}\right)}\right),
$$

where $\mathcal{Q}^{* *}$ is a compact subset of $\mathcal{Q}^{*}$, and $C, \beta$ depend on $K, p, n$ and $\alpha, T$, $\operatorname{dist}\left(\mathcal{Q}, \mathcal{Q}^{*}\right), \operatorname{dist}\left(\mathcal{Q}^{*}, \mathcal{Q}^{* *}\right)$.

We also give the A-B-P comparison principle for the variational inequality A.2 (see Friedman 11. and Tso [25]), which is similar to the comparison principle for the classical solution. Since the solutions for variational inequalities are generally strong solutions rather than classical solutions, the A-B-P comparison theory is more appropriate for variational inequalities. On the other hand, PDEs can be regarded as a special case of variational inequalities if we choose a small enough lower obstacle $\Gamma$ such that the solution $P>\Gamma$ (see Theorem B.1). Hence, the following lemma also applies to PDEs that we considered.

Lemma A.6 For $l=1,2$, let $P^{l}$ be the strong solutions of the following variational inequalities, respectively

$$
\left\{\begin{array}{l}
-\partial_{t} P^{l}-L P^{l}=f^{l} \text { if } P^{l}>\Gamma^{l} \text { and }(t, x) \in \mathcal{Q}_{T}, \\
-\partial_{t} P^{l}-L P^{l} \geq f^{l} \text { if } P^{l}=\Gamma^{l} \text { and }(t, x) \in \mathcal{Q}_{T}, \\
P^{l}=\Psi^{l} \text { on } \partial_{p} \mathcal{Q}_{T} .
\end{array}\right.
$$


Suppose that Assumptions A.1 and A.2 are satisfied, and a satisfies the nonnegative definite condition in $\mathcal{Q}_{T}$, i.e.,

$$
\sum_{i, j=1}^{n} a_{i j}(t, S) \xi_{i} \xi_{j} \geq 0 \text { for any } \xi \in \mathbb{R}^{n},(t, S) \in \mathcal{Q}_{T} .
$$

Moreover, $\Psi^{l}, \Gamma^{l} \in C\left(\overline{\mathcal{Q}}_{T}\right), P^{l} \in W_{p, l o c}^{2,1}\left(\mathcal{Q}_{T}\right) \cap C\left(\overline{\mathcal{Q}}_{T}\right)$ with some $p>n+2$, and there exist a positive constant $C$ and a positive integer $N$ such that

$$
\left|P^{1}\right|+\left|P^{2}\right| \leq C\left(1+|S|^{N}\right) .
$$

Then we have $P^{1} \geq P^{2}$ in $\overline{\mathcal{Q}}_{T}$ if $f^{1} \geq f^{2}, \Psi^{1} \geq \Psi^{2}, \Gamma^{1} \geq \Gamma^{2}$ in $\mathcal{Q}_{T}$.

\section{B Proofs of Propositions}

Proof of Proposition 3.5. It is sufficient to prove the result for $P^{1 b}$, and the proof for $P^{1 s}$ is similar. Without loss of generality, we suppose that $\bar{\kappa}^{*} \leq 1$.

Note that $d_{\mathcal{O}_{1}}\left(v, \mathcal{B}_{t}\right)=\bar{\kappa}^{T} v^{+}$in this case. Then $P^{1 b}$ satisfies

$$
\left\{\begin{array}{l}
-\partial_{t} P^{1 b}-\mathcal{L}_{0} P^{1 b}=\varrho(t)-(\sigma(t) \bar{\kappa})^{T}\left(S D_{S} P^{1 b}\right)^{+} \text {in } \mathcal{N}_{T} \\
P^{1 b}(T, S)=\Psi(S) .
\end{array}\right.
$$

By Proposition 3.4, we have $P^{1 b} \leq P^{0}$. Next, we prove that there exist positive constants $C_{1}, C_{2}$ independent of $\bar{\kappa}$ such that

$$
P^{1 b} \geq P^{0}-\bar{\kappa}^{*} W, \quad W \triangleq C_{1} e^{C_{2}(T-t)}(1+|S|) .
$$

Indeed, since $Q_{l}=\partial_{S_{l}} P^{0}, l=1, \cdots, n$ satisfies

$$
\left\{\begin{array}{l}
-\partial_{t} Q_{l}-\widetilde{\mathcal{L}}^{l} Q_{l}=0 \text { in } \mathcal{N}_{T} \\
Q_{l}(T, S)=\partial_{S_{l}} \Psi(S)
\end{array}\right.
$$

where

$$
\widetilde{\mathcal{L}}^{l} \triangleq \sum_{i, j=1}^{n} \frac{1}{2} a_{i j}(t) S_{i} S_{j} \partial_{S_{i} S_{j}}+\sum_{i=1}^{n}\left[r(t)+a_{l i}(t)\right] S_{i} \partial_{S_{i}} .
$$

By applying Lemma A.6. we can deduce that $\left|\partial_{S_{l}} P^{0}\right| \leq K$, where $K$ is the Lipschitz constant of $\Psi$.

Moreover, it is not difficult to check that

$$
S_{i} \partial_{S_{i}} W=C_{1} e^{C_{2}(T-t)} \frac{S_{i}^{2}}{|S|}, \quad\left|S_{i} \partial_{S_{i}} W\right| \leq W, \quad\left|S_{i} S_{j} \partial_{S_{i} S_{j}} W\right| \leq W .
$$

Hence, we have

$$
\begin{aligned}
& -\partial_{t}\left(P^{0}-\bar{\kappa}^{*} W\right)-\mathcal{L}_{0}\left(P^{0}-\bar{\kappa}^{*} W\right)-\varrho(t)+(\sigma(t) \bar{\kappa})^{T}\left(S D_{S}\left(P^{0}-\bar{\kappa}^{*} W\right)\right)^{+} \\
= & \left(-\partial_{t} P^{0}-\mathcal{L}_{0} P^{0}\right)+\bar{\kappa}^{*}\left(\partial_{t} W+\mathcal{L}_{0} W\right)-\varrho(t)+(\sigma(t) \bar{\kappa})^{T}\left(S D_{S} P^{0}-\bar{\kappa}^{*} S D_{S} W\right)^{+} \\
\leq & -\bar{\kappa}^{*}\left(C_{2} W-C W\right)+C \bar{\kappa}^{*}\left|S D_{S} P^{0}\right| \leq 0
\end{aligned}
$$

provided $C_{1}, C_{2}$ are large enough. Note that the constants are independent of $\bar{\kappa}$.

By applying Lemma A.6 again, we have proved (B.2). By repeating the same argument, we can obtain $P^{1 b} \leq P^{0}+\bar{\kappa}^{*} W$. Hence, if we denote $\Delta P=P^{1 b}-P^{0}$, we have showed that

$$
|\Delta P| \leq \bar{\kappa}^{*} C_{1} e^{C_{2}(T-t)}(1+|S|) .
$$


From PDE (B.1) and Lemma A.4, we have the following estimate

$$
\left\|P^{1 b}\right\|_{W_{p}^{2,1}\left(\mathcal{N}_{T}^{*}\right)} \leq C\left(\|\varrho\|_{L^{p}\left(\mathcal{N}_{T}\right)}+\left\|P^{1 b}\right\|_{L^{p}\left(\mathcal{N}_{T}\right)}\right) \leq \bar{C}
$$

for any compact subset $\mathcal{N}_{T}^{*}$ of $\mathcal{N}_{T}$, where the constants $C, \bar{C}$ depend on $\mathcal{N}_{T}^{*}$, but are independent of $\bar{\kappa}$.

It is clear that $\Delta P$ satisfies

$$
-\partial_{t} \Delta P-\mathcal{L}_{0} \Delta P=-(\sigma(t) \bar{\kappa})^{T}\left(S D_{S} P^{1 b}\right)^{+} \text {in } \mathcal{N}_{T} .
$$

By applying Lemma A.4 again, we deduce that

$$
\|\Delta P\|_{W_{p}^{2,1}\left(\mathcal{Q}_{T}^{*}\right)} \leq C\left(\left\|(\sigma(t) \bar{\kappa})^{T}\left(S D_{S} P^{1 b}\right)^{+}\right\|_{L^{p}\left(\mathcal{Q}_{T}^{*}\right)}+\|\Delta P\|_{L^{p}\left(\mathcal{Q}_{T}^{*}\right)}\right) \leq \bar{C} \bar{\kappa}^{*},
$$

where we have used (B.3) and (B.4).

Proof of Proposition 4.5. First, we prove the existence of the strong solution $P^{b}$ for the variational inequality (4.6).

We use the penalty method to approximate the variational inequality (4.6).

$$
\left\{\begin{array}{l}
-\partial_{t} P_{m}-\mathcal{L}_{0} P_{m}=\varrho(t)-d_{\mathcal{O}}\left((\sigma(t))^{T} S D_{S} P_{m}, \mathcal{B}_{t}\right)+m\left(P_{m}-\Gamma\right)^{-} \text {in } \mathcal{N}_{T} ; \\
P_{m}(T, S)=\Psi(S) .
\end{array}\right.
$$

Since the above problem lies in unbounded domain, and the regularity of the terminal value $\Psi$ is not enough, we need to smooth $\Psi$ and use the following problem in bounded domain to approximate the above PDE in unbounded domain.

$$
\left\{\begin{array}{l}
-\partial_{t} P_{k, m}-\mathcal{L}_{0} P_{k, m}=\varrho(t)-d_{\mathcal{O}}\left((\sigma(t))^{T} S D_{S} P_{k, m}, \mathcal{B}_{t}\right)+m\left(P_{k, m}-\Gamma\right)^{-} \text {in } \mathcal{N}_{T}^{k} ; \\
P_{k, m}(t, S)=\Psi_{k}(S) \text { on } \partial_{p} \mathcal{N}_{T}^{k},
\end{array}\right.
$$

where $\mathcal{N}_{T}^{k} \triangleq[0, T) \times \mathcal{N}^{k}$, and $\mathcal{N}^{k} \triangleq\left\{S \in \mathbb{R}^{n}: 1 / k \leq S_{i} \leq k, i=1, \cdots, n\right\}, k \in \mathbb{N}_{+} . \Psi_{k}$ is the smooth function of $\Psi$, which is defined as follows. Denote by $\eta$ the standard mollifier, then $\eta_{k}(S)=k^{n} \eta(k S)$, and

$$
\Psi_{k}(S)=\int_{\mathbb{R}^{n}} \Psi(y) \eta_{k}(S-y) d y+\frac{K}{k} \text { with } \Psi(y)=0 \text { if } y \notin \mathcal{N} .
$$

It is clear that $\Psi_{k} \in C^{\infty}(\overline{\mathcal{Q}})$ for any $k \in \mathbb{N}_{+}$. It is not difficult to deduce that $\Psi_{k}$ converges to $\Psi$ in $C^{\alpha}\left(\overline{\mathcal{N}^{R}}\right)$ for any $\alpha \in(0,1), R \in \mathbb{N}_{+}$as $k \rightarrow \infty$, and there exists a constant $C$ independent of $m, k$ and $\Gamma$ such that

$$
\left|\Psi_{k}\right| \leq C(1+|S|), \quad\left|D_{S} \Psi_{k}\right| \leq C, \quad \Psi_{k} \geq \Gamma .
$$

Since the nonlinear term $-d_{\mathcal{O}}\left(\sigma(t) v, \mathcal{B}_{t}\right)+m(u-\Gamma)^{-}$is Lipschitz continuous with respect to $u, v$, and $-d_{\mathcal{O}}\left(0, \mathcal{B}_{t}\right)+m(0-\Gamma)^{-}$is bounded in $\mathcal{N}_{T}^{k}$, we deduce that problem (B.6) has a unique strong solution $P_{k, m} \in W_{p}^{2,1}\left(\mathcal{N}_{T}^{k}\right) \cap C\left(\overline{\mathcal{N}_{T}^{k}}\right)$ by Lemma A.3.

Next, we prove some estimates for $P_{k, m}$ which are independent of $k, m$, in order to achieve some proper convergence results. First, we show the upper bound of $P_{k, m}$. More precisely, there exist constants $C_{1}, C_{2}$ independent of $m, k$ such that

$$
P_{k, m} \leq W \triangleq C_{1} e^{C_{2}(T-t)}(1+|S|) .
$$

Indeed, we first choose $C_{1}$ and $C_{2}$ large enough such that $W \geq \Gamma$, then we have

$$
-\partial_{t} W-\mathcal{L}_{0} W-\varrho(t)+d_{\mathcal{O}}\left((\sigma(t))^{T} S D_{S} W, \mathcal{B}_{t}\right)-m(W-\Gamma)^{-} \geq C_{2} W-C W-C \geq 0 .
$$


Moreover, by (B.7), it is clear that

$$
W(t, S) \geq \Psi_{k}(S)=P_{k, m}(t, S) \text { on } \partial_{p} \mathcal{N}_{T}^{k}
$$

provided $C_{1}, C_{2}$ are large enough. Then Lemma A.6 implies (B.8).

Next, we prove the lower bound of $P_{k, m}$, i.e. there exist constants $C_{3}, C_{4}$ independent of $m, k$ and $\Gamma$ such that

$$
P_{k, m} \geq w \triangleq-C_{3} e^{C_{4}(T-t)}(1+|S|) .
$$

Indeed, we can choose $C_{3}$ and $C_{4}$ large enough such that

$$
\begin{aligned}
& -\partial_{t} w-\mathcal{L}_{0} w-\varrho(t)+d_{\mathcal{O}}\left((\sigma(t))^{T} S D_{S} w, \mathcal{B}_{t}\right)-m(w-\Gamma)^{-} \leq C_{4} w-C w+C+C\left|(\sigma(t))^{T} S D_{S} w\right| \leq 0 ; \\
& w(t, S) \leq \Psi_{k}(S)=P_{k, m}(t, S) \text { on } \partial_{p} \mathcal{N}_{T}^{k} .
\end{aligned}
$$

Then Lemma A.6 implies (B.9) .

By applying Lemma A.4 and A.5 to PDE (B.6) in the domain $\mathcal{N}_{T}^{R}$ with $k>R, R \in \mathbb{N}_{+}$, we derive that there exists a constant $C$ depending on $m, R$, but is independent of $k$ such that

$$
\begin{aligned}
& \left\|P_{k, m}\right\|_{W_{p}^{2,1}\left(\mathcal{N}_{T}^{R} \cap\{t \leq T-1 / R\}\right)}+\left\|P_{k, m}\right\|_{C^{\beta, \beta / 2}\left(\overline{\mathcal{N}_{T}^{R}}\right)} \\
\leq & C_{R}\left(\left\|P_{k, m}\right\|_{L^{\infty}\left(\mathcal{N}_{T}^{R}\right)}+\left\|d_{\mathcal{O}}\left(0, \mathcal{B}_{t}\right)\right\|_{L^{p}\left(\mathcal{N}_{T}^{R}\right)}+\left\|m\left(P_{k, m}-\Gamma\right)^{-}\right\|_{L^{p}\left(\mathcal{N}_{T}^{R}\right)}+\left\|\Psi_{k}\right\|_{C^{\alpha}\left(\overline{\mathcal{N}^{R}}\right)}+1\right) \leq C,
\end{aligned}
$$

where we have used (B.8) and (B.9).

Due to the above estimates, we can show that the solution of $(\mathrm{B} .6$ approximates the solution to (B.5) by the method in 28, 27. More precisely, there exists a function $P_{m}$ such that some subsequence of $\left\{P_{k, m}\right\}_{k=1}^{\infty}$ converges to $P_{m}$ weakly in $W_{p}^{2,1}\left(\mathcal{N}_{T}^{R} \cap\{t \leq T-1 / R\}\right)$ and strongly in $C\left(\overline{\mathcal{N}_{T}^{R}}\right)$ for any $m, R \in \mathbb{N}_{+}$, and $P_{m}$ is the strong solution of (B.5). Moreover, by taking $k \rightarrow \infty$ in (B.8) and (B.9), we have

$$
-C_{3} e^{C_{4}(T-t)}(1+|S|) \leq P_{m} \leq C_{1} e^{C_{2}(T-t)}(1+|S|) \text { in } \overline{\mathcal{N}_{T}},
$$

where $C_{1}, C_{2}, C_{3}, C_{4}$ are independent of $m$, and $C_{3}, C_{4}$ are independent of $\Gamma$.

In order to show that the solution of (B.5) approximates the solution of the variational inequality (4.6), we need to prove another lower bound of $P_{m}$ such that

$$
P_{m} \geq \Gamma-\frac{W}{m}, \quad W \triangleq C_{1} e^{C_{2}(T-t)}\left(1+|S|^{N+2}\right)
$$

provided $m$ is large enough, and where $C_{1}, C_{2}$ are constants independent of $m$. Indeed, denote $w^{*}=$ $\Gamma_{1}-W / m$, and we can check that

$$
\begin{aligned}
& -\partial_{t} w^{*}-\mathcal{L}_{0} w^{*}-\varrho(t)+d_{\mathcal{O}}\left((\sigma(t))^{T} S D_{S} w^{*}, \mathcal{B}_{t}\right)-m\left(w^{*}-\Gamma\right)^{-} \\
\leq & \left(-\partial_{t} \Gamma_{1}-\mathcal{L}_{0} \Gamma_{1}\right)-\frac{C_{2} W-C W}{m}+C+C\left|(\sigma(t))^{T} S D_{S} \Gamma_{1}\right|-W \leq C\left(1+|S|^{N+2}\right)-W \leq 0
\end{aligned}
$$

provided $C_{1}, C_{2}$ are large enough. Then Lemma A.6 implies $P_{m} \geq \Gamma_{1}-W / m$. By repeating the same argument, we can deduce that $P_{k, m} \geq \Gamma_{2}-W / m$, so (B.11) is obvious.

By applying Lemma A.4 and A.5. we derive that there exists a constant $C_{R}$ independent of $m$ such that

$$
\begin{aligned}
& \left\|P_{m}\right\|_{W_{p}^{2,1}\left(\mathcal{N}_{T}^{R} \cap\{t \leq T-1 / R\}\right)}+\left\|P_{m}\right\|_{C^{\beta, \beta / 2}\left(\overline{\mathcal{N}_{T}^{R}}\right)} \\
\leq & C_{R}\left(\left\|P_{m}\right\|_{L^{\infty}\left(\mathcal{N}_{T}^{R}\right)}+\left\|d_{\mathcal{O}}\left(0, \mathcal{B}_{t}\right)\right\|_{L^{p}\left(\mathcal{N}_{T}^{R}\right)}+\left\|m\left(P_{m}-\Gamma\right)^{-}\right\|_{L^{p}\left(\mathcal{N}_{T}^{R}\right)}+\|\Psi\|_{C^{\alpha}\left(\overline{\mathcal{N}^{R}}\right)}+1\right) \leq C,
\end{aligned}
$$


where we have used (B.10) and (B.11), and the constant $C$ is independent of $m$.

Thanks to the above estimates, we can show that the solution of (B.5) approximates the solution of (4.6) by the method in 11, 28. More precisely, there exists a function $P$ such that some subsequence of $\left\{P_{m}\right\}_{m=1}^{\infty}$ converges to $P$ weakly in $W_{p}^{2,1}\left(\mathcal{N}_{T}^{R} \cap\{t \leq T-1 / R\}\right)$ and strongly in $C\left(\overline{\mathcal{N}_{T}^{R}}\right)$ for any $R \in \mathbb{N}_{+}$, and $P$ is the strong solution of Problem (4.6). Therefore, we have proved the existence of the strong solution $P^{b}$ for the variational inequality (4.6).

Since $R$ is arbitrary, we deduce that $P^{b} \in W_{p, l o c}^{2,1}\left(\mathcal{N}_{T}\right) \cap C\left(\overline{\mathcal{N}_{T}}\right)$. Moreover, by taking $m \rightarrow \infty$ in (B.10), we have

$$
-C_{3} e^{C_{4}(T-t)}(1+|S|) \leq P^{b} \leq C_{1} e^{C_{2}(T-t)}(1+|S|) \text { in } \overline{\mathcal{N}_{T}},
$$

where $C_{3}, C_{4}$ are independent of $\Gamma$. The uniqueness of the strong solution $P^{b}$ for the variational inequality (4.6) follows from Lemma A.6. The proof of the results for $P^{s}$ is similar.

Proof of Proposition 4.8. The proof is similar to that of Proposition 3.5. First, we prove the result for $P^{1 b}$. Without loss of generality, we suppose that $\bar{\kappa}^{*} \leq 1$.

We first prove that

$$
\left|\partial_{S_{l}} P^{0}\right| \leq K \text { for any } l=1, \cdots, n,
$$

where $K$ is the Lipschitz constant of $\Gamma$ and $\Psi$.

We must start from the penalty problem (B.5) to prove the result because the regularity of the solution for the variational inequality (4.6) is not enough. Note that $d_{\mathcal{O}_{0}}\left(v, \mathcal{B}_{t}\right)=0$ in this case, then the penalty problem is

$$
\left\{\begin{array}{l}
-\partial_{t} P_{m}^{0}-\mathcal{L}_{0} P_{m}^{0}=\varrho(t)+m\left(P_{m}^{0}-\Gamma\right)^{-} ; \\
P_{m}^{0}(T, S)=\Psi(S) .
\end{array}\right.
$$

Denote $Q_{l}=\partial_{S_{l}} P_{m}^{0}, l=1, \cdots, n$ which satisfies

$$
\left\{\begin{array}{l}
-\partial_{t} Q_{l}-\widehat{\mathcal{L}}^{l} Q_{l}=m \partial_{S_{l}} \Gamma I_{\left\{P_{m}^{0}<\Gamma\right\}} \text { in } \mathcal{N}_{T} ; \\
Q_{l}(T, S)=\partial_{S_{l}} \Psi(S),
\end{array}\right.
$$

where

$$
\widehat{\mathcal{L}}^{l} \triangleq \sum_{i, j=1}^{n} \frac{1}{2} a_{i j}(t) S_{i} S_{j} \partial_{S_{i} S_{j}}+\sum_{i=1}^{n}\left[r(t)+a_{l i}(t)\right] S_{i} \partial_{S_{i}}-m I_{\left\{P_{m}^{0}<\Gamma\right\}} .
$$

By applying Lemma A.6, we have

$$
\left|\partial_{S_{l}} P_{m}^{0}\right| \leq K \text { for any } l=1, \cdots, n .
$$

By letting $m \rightarrow \infty$, we deduce (B.13).

Note that $d_{\mathcal{O}_{1}}\left(v, \mathcal{B}_{t}\right)=\bar{\kappa}^{T} v^{+}$in this case. Then $P^{1 b}$ satisfies

$$
\left\{\begin{array}{l}
-\partial_{t} P^{1 b}-\mathcal{L}_{0} P^{1 b}=\varrho(t)-(\sigma(t) \bar{\kappa})^{T}\left(S D_{S} P^{1 b}\right)^{+} \text {if } P^{1 b}>\Gamma ; \\
-\partial_{t} P^{1 b}-\mathcal{L}_{0} P^{1 b} \geq \varrho(t)-(\sigma(t) \bar{\kappa})^{T}\left(S D_{S} P^{1 b}\right)^{+} \text {if } P^{1 b}=\Gamma ; \\
P^{1 b}(T, S)=\Psi(S),
\end{array}\right.
$$

From Proposition 4.7 we have $P^{1 b} \leq P^{0}$. Next, we prove that there exist positive constants $C_{1}, C_{2}$ independent of $\bar{\kappa}$ such that

$$
P^{1 b} \geq w^{*}=P^{0}-\bar{\kappa}^{*} W, \quad W \triangleq C_{1} e^{C_{2}(T-t)}(1+|S|) .
$$


Indeed, repeating the same argument as in the proof of Proposition 3.5 we have

$$
\begin{aligned}
& \widetilde{\varrho}(t) \triangleq\left(-\partial_{t} w^{*}-\mathcal{L}_{0} w^{*}+(\sigma(t) \bar{\kappa})^{T}\left(S D_{S} w^{*}\right)^{+}\right)-\left(-\partial_{t} P^{0}-\mathcal{L}_{0} P^{0}\right)+\varrho(t) \\
= & \bar{\kappa}^{*}\left(\partial_{t} W+\mathcal{L}_{0} W\right)+(\sigma(t) \bar{\kappa})^{T}\left(S D_{S} P^{0}-\bar{\kappa}^{*} S D_{S} W\right)^{+}+\varrho(t) \\
\leq & -\bar{\kappa}^{*}\left(C_{2} W-C W\right)+C \bar{\kappa}^{*}\left|S D_{S} P^{0}\right|+\varrho(t) \leq \varrho(t)
\end{aligned}
$$

provided $C_{1}, C_{2}$ are large enough, where we have used (B.13).

Hence, the variational inequality for $P^{0}$ implies that $w^{*}$ satisfies

$$
\left\{\begin{array}{l}
-\partial_{t} w^{*}-\mathcal{L}_{0} w^{*}+(\sigma(t) \bar{\kappa})^{T}\left(S D_{S} w^{*}\right)^{+}=\widetilde{\varrho}(t) \text { if } w^{*}>\Gamma-\bar{\kappa}^{*} W \\
-\partial_{t} w^{*}-\mathcal{L}_{0} w^{*}+(\sigma(t) \bar{\kappa})^{T}\left(S D_{S} w^{*}\right)^{+} \geq \widetilde{\varrho}(t) \text { if } w^{*}=\Gamma-\bar{\kappa}^{*} W ; \\
w^{*}(T, S)=\Psi(S)-\bar{\kappa}^{*} W(T, S) .
\end{array}\right.
$$

By applying Lemma A.6 again, we have (B.15), so the result for $P^{1 b}$ has been proved.

Next, we prove the result for $P^{1 s}$. Since $P^{1 s}=\Gamma=P^{1 b}$ in the set $\left\{P^{1 b}=\Gamma\right\}$, the result for $P^{1 b}$ implies that

$$
\left|P^{1 s}-P^{0}\right| \leq C \bar{\kappa}^{*} \text { in }\left\{P^{1 b}=\Gamma\right\} .
$$

Then it is sufficient to prove that the above inequality holds in the set $\left\{P^{1 b}>\Gamma\right\}$.

From Proposition 4.7 we have $P^{1 b} \leq P^{0}$, so that

$$
\left\{P^{1 b}>\Gamma\right\} \subset\left\{P^{0}>\Gamma\right\} .
$$

Hence, in the set $\left\{P^{1 b}>\Gamma\right\}, P^{1 s}$ and $P^{0}$ satisfy

$$
\left\{\begin{array}{l}
-\partial_{t} P^{1 s}-\mathcal{L}_{0} P^{1 s}=\varrho(t)+(\sigma(t) \bar{\kappa})^{T}\left(S D_{S} P^{1 s}\right)^{-} \text {in }\left\{P^{1 b}>\Gamma\right\} ; \\
-\partial_{t} P^{0}-\mathcal{L}_{0} P^{0}=\varrho(t) \text { in }\left\{P^{1 b}>\Gamma\right\} ; \\
\left|P^{1 s}-P^{0}\right|=\left|P^{1 b}-P^{0}\right| \leq C \bar{\kappa}^{*}(1+|S|) \text { on } \partial_{p}\left\{P^{1 b}>\Gamma\right\} .
\end{array}\right.
$$

Repeating the same argument as above, we have proved the result for $P^{1 s}$.

To finish the Appendix, we give the following connection between the variational inequality (4.6) and PDEs (3.4), which is a direct consequence of (B.12).

Theorem B.1 Suppose that Assumptions 2.1. 2.2, 3.1 are satisfied. Then there exist some functions $\Gamma \in C^{\infty}\left(\mathcal{N}_{T}\right)$ satisfying Assumption 4.1. such that (4.6) is equivalent to 3.4).

Proof. From (B.12) and Assumption 3.1, we can choose a large enough constant $C$ such that

$$
\Gamma=-2 C \sqrt{1+|S|^{2}}
$$

which satisfies Assumption 4.1, and $\Gamma \in C^{\infty}\left(\mathcal{N}_{T}\right)$ with $P^{b}>\Gamma$. Since $P^{b}>\Gamma$, then (4.6) implies that

$$
-\partial_{t} P^{b}-\mathcal{L}_{0} P^{b}=\varrho(t)-d_{\mathcal{O}}\left((\sigma(t))^{T} S D_{S} P^{b}, \mathcal{B}_{t}\right) \text { in } \mathcal{N}_{T} .
$$

Hence, (4.6) is equivalent to (3.4). 


\section{References}

[1] Anderson E, Hansen L, Sargent T. Robustness, detection and the price of risk. Technical Report, University of Chicago, 1999

[2] Ankirchner S, Imkeller P, dos Reis G. Pricing and hedging of derivatives based on non-tradable underlyings. Mathematical Finance, 20(2): 289-312 (2010)

[3] Becherer D. Rational hedging and valuation of integrated risks under constant absolute risk aversion. Insurance: Mathematics and Economics, 33(1): 1-28 (2003)

[4] Chen, Z, Epstein L. Ambiguity, risk and asset return in continuous time. Econometrica, 70: 1403-1443 (2002)

[5] Cheridito P, Hu Y. Optimal consumption and investment in incomplete markets with general constraints. Stoch. Dyn., 11 283: 283-299 (2011)

[6] Cont R. Model uncertainty and its impact on the pricing of derivative instruments. Mathematical Finance, 16(3): 519-547 (2006)

[7] Davis M. Optimal hedging with basis risk, Second Bachelier Colloquium on Stochastic Calculus and Probability, edited by Kabanov Y, Lipster R, Stoyanov J, Springer: 169-187 (2006).

[8] Duffie D, Epstein L. Stochastic differential utility. Econometrica, 60: 353-394 (1992)

[9] El Karoui N, Kapoudjian C, Pardoux E, Peng S, Quenez M C. Reflected solutions of backward SDEs, and related obstacle problems for PDEs. Annals of Probability, 25: $702-737$ (1997)

[10] El Karoui N, Peng S, Quenez M C. Backward stochastic differential equations in finance. Mathematical Finance, 7(1): 1-71 (1997)

[11] Friedman A. Variational principles and free-boundary problems. John Wiley \& Sons, New York: 1982

[12] Guo D, Song B, Wang S. Backward stochastic differential equations and nonlinear pricing Parisian (Parasian) options. Scientia China Mathematics, 43: 91-103 (2013)

[13] Henderson V. Valuation of claims on nontraded assets using utility maximization. Mathematical Finance, 12: $351-373(2002)$

[14] Henderson V. and Liang G. Pseudo linear pricing rule for utility indifference valuation. Finance and Stochastics, to appear.

[15] Hodges S, Neuberger A. Optimal replication of contingent claims under transaction costs. Review of Future Markets, 8: 222-239 (1989)

[16] Hu Y, Imkeller P, Müller M. Utility maximization in incomplete markets. Annals of Applied Probability, 15: 1691-1712 (2005)

[17] Jaimungal S, Sigloch G. Incorporating risk and ambiguity aversion into a hybrid model of default. Mathematical Finance, 22(1): 57-81 (2012)

[18] Jiang L. Mathematical modelling and methods of option pricing. Singapore: World Scientific, 2005

[19] Karatzas I, Shreve S. Methods of mathematical finance. New York: Springer, 1998

[20] Ladyzenskaja O A, Solonnikov V A, Ural'ceva N M. Linear and quasi-linear equations of parabolic type. Providence: American Math. Soc., 1968

[21] Lieberman G M. Second order parabolic differential equations. Singapore: World Scientific, 1996

[22] Mania M, Schweizer M. Dynamic exponential utility indifference valuation. Ann. Appl. Probab., 15: 2113$2143(2005)$

[23] Musiela M, Zariphopoulou T. An example of indifference prices under exponential preferences. Finance and Stochastics, 8: 229-239 (2004)

[24] Sircar R, Zariphopoulou T. Bounds and asymptotic approximations for utility prices when volatility is random. SIAM J. Control Optim., 43(4): 1328-1353 (2005) 
[25] Tao K. On an Aleksandrov-Bakel'Man type maximum principle for second-order parabolic equations. Communications in Partial Differential Equations, 10(5): 543-553 (1985)

[26] Yang Z, Tang S. Dynkin game of stochastic differential equations with random coefficients, and associated backward stochastic partial differential variational inequality. SIAM J. Control Optim., 51: 64-95 (2013)

[27] Yi F, Yang Z. A Variational Inequality Arising from European Option Pricing with Transaction Costs. Science in China Ser. A: Mathematics, 51: 935-954 (2008)

[28] Yi F, Yang Z, Wang X. A variational inequality arising from European installment call options pricing. SIAM on Mathematical Analysis, 40, 306-326 (2008) 